Attachments 


\section{PuSGeN catalogue}

\begin{tabular}{|c|c|c|c|c|c|c|c|c|c|}
\hline \multirow{2}{*}{ No. } & \multirow{2}{*}{ Long( $\left(^{\circ}\right)$} & \multirow{2}{*}{$\operatorname{Lat}\left({ }^{0}\right)$} & \multicolumn{3}{|c|}{ Date } & \multirow{2}{*}{$\begin{array}{l}\text { Mag. } \\
\left(\mathbf{M}_{w}\right)\end{array}$} & \multirow{2}{*}{ depth (km) } & \multicolumn{2}{|c|}{ time } \\
\hline & & & dt & mn & $\mathbf{y r}$ & & & hr & mn \\
\hline 1 & 94,648 & 6,843 & 8 & 11 & 2015 & 6,6 & 10 & 16 & 47 \\
\hline 2 & 94,534 & 6,984 & 8 & 11 & 2015 & 5,6 & 10 & 19 & 14 \\
\hline 3 & 94,802 & 6,408 & 9 & 11 & 2015 & 5,5 & 10 & 8 & 12 \\
\hline 4 & 94,883 & 6,518 & 9 & 11 & 2015 & 5,43773 & 11,83 & 6 & 12 \\
\hline 5 & 94,532 & 6,833 & 14 & 11 & 2015 & 5,43773 & 10 & 16 & 53 \\
\hline 6 & 94,762 & 6,934 & 8 & 11 & 2015 & 5,33752 & 10 & 16 & 59 \\
\hline 7 & 94,487 & 6,853 & 8 & 11 & 2015 & 5,03689 & 10 & 16 & 42 \\
\hline 8 & 94,634 & 6,538 & 9 & 11 & 2015 & 4,93668 & 12,58 & 7 & 7 \\
\hline 9 & 94,476 & 6,911 & 8 & 11 & 2015 & 4,93668 & 10 & 20 & 59 \\
\hline 10 & 94,642 & 6,581 & 9 & 11 & 2015 & 4,83647 & 10 & 7 & 2 \\
\hline 11 & 94,796 & 6,627 & 9 & 11 & 2015 & 4,83647 & 10 & 7 & 28 \\
\hline 12 & 94,762 & 6,77 & 8 & 11 & 2015 & 4,83647 & 10 & 17 & 46 \\
\hline 13 & 94,626 & 7,127 & 8 & 11 & 2015 & 4,83647 & 10 & 20 & 30 \\
\hline 14 & 94,403 & 7,262 & 31 & 12 & 2015 & 4,83647 & 35 & 3 & 23 \\
\hline 15 & 94,725 & 6,505 & 9 & 11 & 2015 & 4,8 & 10 & 7 & 13 \\
\hline 16 & 94,828 & 6,638 & 9 & 11 & 2015 & 4,73626 & 10 & 7 & 5 \\
\hline 17 & 94,349 & 6,672 & 15 & 11 & 2015 & 4,73626 & 10 & 13 & 7 \\
\hline 18 & 94,475 & 6,861 & 8 & 11 & 2015 & 4,73626 & 10 & 18 & 48 \\
\hline 19 & 94,663 & 6,321 & 10 & 11 & 2015 & 4,63605 & 10 & 17 & 22 \\
\hline 20 & 94,581 & 6,784 & 8 & 11 & 2015 & 4,63605 & 10 & 19 & 10 \\
\hline 21 & 94,587 & 6,865 & 8 & 11 & 2015 & 4,63605 & 10 & 16 & 29 \\
\hline 22 & 94,489 & 6,878 & 10 & 11 & 2015 & 4,63605 & 10 & 15 & 59 \\
\hline 23 & 94,543 & 6,931 & 12 & 11 & 2015 & 4,63605 & 10 & 22 & 34 \\
\hline 24 & 94,272 & 6,991 & 15 & 11 & 2015 & 4,63605 & 9,16 & 14 & 49 \\
\hline 25 & 94,231 & 7,593 & 21 & 3 & 2014 & 6,4 & 24,81 & 13 & 41 \\
\hline 26 & 94,36 & 7,452 & 18 & 11 & 2014 & 5,6 & 6,71 & 3 & 25 \\
\hline 27 & 94,223 & 7,508 & 21 & 3 & 2014 & 5,53794 & 14,38 & 14 & 11 \\
\hline 28 & 94,29 & 7,548 & 21 & 3 & 2014 & 5,33752 & 11,61 & 14 & 25 \\
\hline 29 & 94,315 & 7,593 & 11 & 11 & 2014 & 5,3 & 16,25 & 7 & 50 \\
\hline 30 & 94,364 & 7,389 & 6 & 11 & 2014 & 5,23731 & 12,47 & 15 & 46 \\
\hline 31 & 94,275 & 7,436 & 21 & 3 & 2014 & 5,23731 & 15,29 & 21 & 3 \\
\hline 32 & 94,174 & 7,323 & 21 & 3 & 2014 & 5,23731 & 10 & 14 & 13 \\
\hline 33 & 94,305 & 7,444 & 11 & 11 & 2014 & 5,1371 & 28,15 & 8 & 4 \\
\hline 34 & 94,722 & 7,039 & 8 & 11 & 2015 & 5,03689 & 20,48 & 10 & 53 \\
\hline 35 & 94,705 & 7,769 & 21 & 3 & 2014 & 5,03689 & 10 & 13 & 54 \\
\hline 36 & 94,884 & 7,136 & 8 & 11 & 2015 & 4,93668 & 10 & 11 & 42 \\
\hline 37 & 94,675 & 7,433 & 26 & 10 & 2014 & 4,93668 & 17,35 & 2 & 58 \\
\hline 38 & 94,643 & 7,393 & 20 & 11 & 2014 & 4,93668 & 23,6 & 6 & 5 \\
\hline
\end{tabular}




\begin{tabular}{|c|c|c|c|c|c|c|c|c|c|}
\hline \multirow{2}{*}{ No. } & \multirow{2}{*}{$\operatorname{Long}\left({ }^{\circ}\right)$} & \multirow{2}{*}{$\operatorname{Lat}\left({ }^{0}\right)$} & \multicolumn{3}{|c|}{ Date } & \multirow{2}{*}{$\begin{array}{l}\text { Mag. } \\
\left(\mathbf{M}_{\mathrm{w}}\right)\end{array}$} & \multirow{2}{*}{ depth $(\mathbf{k m})$} & \multicolumn{2}{|c|}{ time } \\
\hline & & & dt & mn & $\mathrm{yr}$ & & & hr & $\mathbf{m n}$ \\
\hline 39 & 94,407 & 7,411 & 18 & 11 & 2014 & 4,93668 & 7,77 & 6 & 12 \\
\hline 40 & 94,407 & 7,614 & 21 & 3 & 2014 & 4,93668 & 10 & 13 & 52 \\
\hline 41 & 94,406 & 7,385 & 18 & 11 & 2014 & 4,93668 & 15,35 & 6 & 6 \\
\hline 42 & 94,386 & 7,358 & 14 & 10 & 2014 & 4,93668 & 3,36 & 18 & 36 \\
\hline 43 & 94,322 & 7,557 & 21 & 3 & 2014 & 4,93668 & 10 & 14 & 18 \\
\hline 44 & 94,308 & 7,321 & 21 & 3 & 2014 & 4,93668 & 10 & 14 & 26 \\
\hline 45 & 94,273 & 7,453 & 19 & 12 & 2014 & 4,93668 & 23,4 & 2 & 36 \\
\hline 46 & 94,271 & 7,79 & 21 & 3 & 2014 & 4,93668 & 10 & 14 & 16 \\
\hline 47 & 94,232 & 7,788 & 21 & 3 & 2014 & 4,93668 & 10 & 14 & 49 \\
\hline 48 & 94,206 & 7,283 & 21 & 3 & 2014 & 4,93668 & 10 & 13 & 56 \\
\hline 49 & 94,105 & 7,603 & 21 & 3 & 2014 & 4,93668 & 12,22 & 14 & 45 \\
\hline 50 & 94,065 & 7,731 & 21 & 3 & 2014 & 4,93668 & 7,06 & 15 & 48 \\
\hline 51 & 94,03 & 7,382 & 21 & 3 & 2014 & 4,93668 & 10 & 14 & 28 \\
\hline 52 & 94,771 & 7,145 & 8 & 11 & 2015 & 4,83647 & 10 & 10 & 7 \\
\hline 53 & 94,717 & 7,436 & 9 & 10 & 2014 & 4,83647 & 15,35 & 20 & 34 \\
\hline 54 & 94,567 & 7,412 & 17 & 10 & 2014 & 4,83647 & 38,72 & 23 & 32 \\
\hline 55 & 94,48 & 7,998 & 21 & 3 & 2014 & 4,83647 & 10 & 14 & 3 \\
\hline 56 & 94,447 & 7,597 & 17 & 11 & 2014 & 4,83647 & 24,18 & 5 & 8 \\
\hline 57 & 94,406 & 7,66 & 18 & 10 & 2014 & 4,83647 & 10 & 9 & 47 \\
\hline 58 & 94,397 & 7,463 & 21 & 3 & 2014 & 4,83647 & 10 & 14 & 47 \\
\hline 59 & 94,379 & 7,382 & 22 & 3 & 2014 & 4,83647 & 8,66 & 0 & 10 \\
\hline 60 & 94,311 & 7,541 & 21 & 3 & 2014 & 4,83647 & 10 & 13 & 50 \\
\hline 61 & 94,304 & 7,392 & 13 & 10 & 2014 & 4,83647 & 23,06 & 17 & 21 \\
\hline 62 & 94,262 & 7,503 & 21 & 3 & 2014 & 4,83647 & 10 & 15 & 8 \\
\hline 63 & 94,244 & 7,523 & 21 & 3 & 2014 & 4,83647 & 10 & 15 & 23 \\
\hline 64 & 94,756 & 7,102 & 8 & 11 & 2015 & 4,73626 & 10 & 14 & 54 \\
\hline 65 & 94,71 & 7,754 & 21 & 3 & 2014 & 4,73626 & 10,03 & 21 & 11 \\
\hline 66 & 94,59 & 7,617 & 18 & 10 & 2014 & 4,73626 & 41,02 & 10 & 10 \\
\hline 67 & 94,553 & 7,745 & 25 & 3 & 2014 & 4,73626 & 10,21 & 20 & 46 \\
\hline 68 & 94,523 & 7,66 & 18 & 10 & 2014 & 4,73626 & 45,91 & 9 & 57 \\
\hline 69 & 94,478 & 7,509 & 24 & 10 & 2014 & 4,73626 & 27,64 & 17 & 23 \\
\hline 70 & 94,447 & 7,038 & 21 & 3 & 2014 & 4,73626 & 10 & 13 & 53 \\
\hline 71 & 94,374 & 7,46 & 26 & 12 & 2014 & 4,73626 & 41,59 & 20 & 38 \\
\hline 72 & 94,346 & 7,471 & 2 & 11 & 2014 & 4,73626 & 29,8 & 11 & 38 \\
\hline 73 & 94,308 & 7,485 & 26 & 10 & 2014 & 4,73626 & 48,94 & 22 & 7 \\
\hline 74 & 94,258 & 7,52 & 21 & 3 & 2014 & 4,73626 & 11,42 & 18 & 8 \\
\hline 75 & 94,253 & 7,372 & 21 & 3 & 2014 & 4,73626 & 7,53 & 23 & 50 \\
\hline 76 & 94,233 & 7,483 & 21 & 3 & 2014 & 4,73626 & 14,93 & 14 & 17 \\
\hline 77 & 94,2 & 7,322 & 21 & 3 & 2014 & 4,73626 & 10,93 & 18 & 50 \\
\hline 78 & 94,182 & 7,352 & 21 & 3 & 2014 & 4,73626 & 10 & 15 & 10 \\
\hline 79 & 94,169 & 7,233 & 21 & 3 & 2014 & 4,73626 & 10 & 14 & 5 \\
\hline
\end{tabular}




\begin{tabular}{|c|c|c|c|c|c|c|c|c|c|}
\hline \multirow{2}{*}{ No. } & \multirow{2}{*}{$\operatorname{Long}\left({ }^{\circ}\right)$} & \multirow{2}{*}{$\operatorname{Lat}\left({ }^{0}\right)$} & \multicolumn{3}{|c|}{ Date } & \multirow{2}{*}{$\begin{array}{l}\text { Mag. } \\
\left(\mathbf{M}_{\mathrm{w}}\right)\end{array}$} & \multirow{2}{*}{ depth $(\mathbf{k m})$} & \multicolumn{2}{|c|}{ time } \\
\hline & & & dt & $\mathbf{m n}$ & yr & & & hr & $\mathbf{m n}$ \\
\hline 80 & 94,129 & 7,733 & 21 & 3 & 2014 & 4,73626 & 2,48 & 15 & 43 \\
\hline 81 & 94,082 & 7,426 & 21 & 3 & 2014 & 4,73626 & 10 & 13 & 59 \\
\hline 82 & 94,686 & 7,55 & 6 & 11 & 2014 & 4,63605 & 19,06 & 22 & 0 \\
\hline 83 & 94,604 & 7,606 & 21 & 3 & 2014 & 4,63605 & 10 & 14 & 42 \\
\hline 84 & 94,599 & 7,715 & 21 & 3 & 2014 & 4,63605 & 10 & 15 & 9 \\
\hline 85 & 94,595 & 7,519 & 26 & 10 & 2014 & 4,63605 & 12,61 & 2 & 56 \\
\hline 86 & 94,499 & 7,559 & 21 & 3 & 2014 & 4,63605 & 10 & 15 & 24 \\
\hline 87 & 94,491 & 7,493 & 8 & 10 & 2014 & 4,63605 & 16,19 & 11 & 6 \\
\hline 88 & 94,476 & 7,548 & 21 & 3 & 2014 & 4,63605 & 11,72 & 17 & 5 \\
\hline 89 & 94,443 & 7,526 & 14 & 10 & 2014 & 4,63605 & 37,18 & 3 & 32 \\
\hline 90 & 94,442 & 7,248 & 22 & 11 & 2014 & 4,63605 & 35 & 10 & 49 \\
\hline 91 & 94,377 & 7,397 & 11 & 10 & 2014 & 4,63605 & 35 & 16 & 9 \\
\hline 92 & 94,326 & 7,328 & 11 & 10 & 2014 & 4,63605 & 27,7 & 20 & 36 \\
\hline 93 & 94,321 & 7,659 & 15 & 5 & 2014 & 4,63605 & 10 & 17 & 56 \\
\hline 94 & 94,31 & 7,377 & 14 & 11 & 2014 & 4,63605 & 10 & 21 & 19 \\
\hline 95 & 94,303 & 7,394 & 2 & 11 & 2014 & 4,63605 & 30,29 & 0 & 58 \\
\hline 96 & 94,273 & 7,573 & 21 & 3 & 2014 & 4,63605 & 7,8 & 14 & 36 \\
\hline 97 & 94,231 & 7,618 & 24 & 10 & 2014 & 4,63605 & 45,42 & 15 & 26 \\
\hline 98 & 94,215 & 7,112 & 30 & 8 & 2014 & 4,63605 & 12,85 & 3 & 35 \\
\hline 99 & 94,207 & 7,408 & 1 & 11 & 2014 & 4,63605 & 10 & 0 & 11 \\
\hline 100 & 94,207 & 7,382 & 21 & 3 & 2014 & 4,63605 & 10 & 14 & 53 \\
\hline 101 & 94,189 & 7,301 & 4 & 11 & 2014 & 4,63605 & 10 & 5 & 36 \\
\hline 102 & 94,181 & 7,221 & 9 & 11 & 2014 & 4,63605 & 10 & 11 & 43 \\
\hline 103 & 94,138 & 7,264 & 28 & 10 & 2014 & 4,63605 & 38,7 & 16 & 22 \\
\hline 104 & 94,107 & 7,428 & 11 & 11 & 2014 & 4,63605 & 10,88 & 15 & 24 \\
\hline 105 & 95,956 & 4,794 & 21 & 1 & 2013 & 6,1 & 9,58 & 22 & 22 \\
\hline 106 & 95,838 & 4,986 & 22 & 10 & 2013 & 5,4 & 14,64 & 5 & 40 \\
\hline 107 & 96,427 & 4,492 & 14 & 3 & 2013 & 4,9 & 8,9 & 18 & 18 \\
\hline 108 & 96,015 & 5,019 & 21 & 1 & 2013 & 4,83647 & 10 & 22 & 48 \\
\hline 109 & 95,999 & 5,082 & 22 & 10 & 2013 & 4,73626 & 45,89 & 14 & 16 \\
\hline 110 & 95,745 & 4,793 & 28 & 12 & 2013 & 4,73626 & 9,9 & 15 & 25 \\
\hline 111 & 95,978 & 4,969 & 11 & 2 & 2013 & 4,7 & 0,38 & 1 & 2 \\
\hline 112 & 95,698 & 5,233 & 22 & 8 & 2013 & 4,7 & 5,17 & 23 & 59 \\
\hline 113 & 95,897 & 5,056 & 18 & 2 & 2013 & 4,63605 & 10 & 15 & 22 \\
\hline 114 & 95,89 & 4,919 & 25 & 1 & 2013 & 4,63605 & 10 & 5 & 0 \\
\hline 115 & 95,801 & 5,037 & 27 & 1 & 2013 & 4,6 & 9,63 & 14 & 24 \\
\hline 116 & 96,554 & 4,415 & 1 & 8 & 2014 & 5,03689 & 5,08 & 3 & 10 \\
\hline 117 & 96,536 & 4,407 & 5 & 6 & 2014 & 4,83647 & 1,2 & 8 & 11 \\
\hline 118 & 96,104 & 4,88 & 8 & 7 & 2014 & 4,83647 & 10,84 & 17 & 31 \\
\hline 119 & 96,038 & 4,909 & 27 & 6 & 2014 & 4,73626 & 8,6 & 7 & 35 \\
\hline 120 & 95,879 & 4,88 & 9 & 5 & 2014 & 4,73626 & 36,89 & 20 & 0 \\
\hline
\end{tabular}




\begin{tabular}{|c|c|c|c|c|c|c|c|c|c|}
\hline \multirow{2}{*}{ No. } & \multirow{2}{*}{$\operatorname{Long}\left({ }^{\circ}\right)$} & \multirow{2}{*}{$\operatorname{Lat}\left(^{0}\right)$} & \multicolumn{3}{|c|}{ Date } & \multirow{2}{*}{$\begin{array}{l}\text { Mag. } \\
\left(\mathbf{M}_{\mathbf{w}}\right)\end{array}$} & \multirow{2}{*}{ depth $(\mathbf{k m})$} & \multicolumn{2}{|c|}{ time } \\
\hline & & & dt & $\mathbf{m n}$ & $\mathrm{yr}$ & & & hr & mn \\
\hline 121 & 96,309 & 4,67 & 27 & 5 & 2014 & 4,63605 & 23,48 & 6 & 49 \\
\hline 122 & 96,113 & 4,835 & 2 & 3 & 2015 & 5,1371 & 3,85 & 10 & 33 \\
\hline 123 & 95,962 & 4,852 & 2 & 3 & 2015 & 5,03689 & 21,38 & 14 & 19 \\
\hline 124 & 96,097 & 4,882 & 27 & 3 & 2015 & 4,73626 & 18,14 & 20 & 25 \\
\hline 125 & 99,147 & 1,64 & 19 & 5 & 2008 & 6 & 10 & 14 & 26 \\
\hline 126 & 99,04 & 1,41 & 19 & 5 & 2008 & 5,3 & 10 & 18 & 9 \\
\hline 127 & 99,05 & 2,01 & 3 & 10 & 2008 & 5,3 & 35 & 14 & 7 \\
\hline 128 & 99,06 & 1,5 & 1 & 6 & 2008 & 5,3 & 35 & 19 & 48 \\
\hline 129 & 99,08 & 1,75 & 30 & 11 & 2008 & 5,3 & 21,4 & 21 & 19 \\
\hline 130 & 99,18 & 1,724 & 19 & 5 & 2008 & 5,3 & 10 & 14 & 49 \\
\hline 131 & 99,03 & 1,79 & 16 & 11 & 2008 & 5,2 & 35 & 10 & 51 \\
\hline 132 & 99,04 & 1,49 & 19 & 5 & 2008 & 5,2 & 10 & 18 & 25 \\
\hline 133 & 99,15 & 1,74 & 7 & 6 & 2008 & 5,2 & 35 & 0 & 7 \\
\hline 134 & 99,21 & 1,41 & 19 & 5 & 2008 & 5,2 & 10 & 20 & 17 \\
\hline 135 & 98,957 & 1,34 & 19 & 5 & 2008 & 5,03689 & 10 & 14 & 30 \\
\hline 136 & 99,165 & 1,754 & 22 & 5 & 2008 & 5,03689 & 40,8 & 18 & 58 \\
\hline 137 & 98,74 & 2,059 & 28 & 2 & 2010 & 5 & 10,81 & 12 & 13 \\
\hline 138 & 99,161 & 1,872 & 9 & 4 & 2010 & 4,9 & 1,19 & 6 & 29 \\
\hline 139 & 99,19 & 1,663 & 22 & 5 & 2008 & 4,63605 & 35 & 17 & 55 \\
\hline 140 & 100,498 & $-0,493$ & 6 & 3 & 2007 & 6,4 & 19 & 3 & 49 \\
\hline 141 & 100,53 & $-0,488$ & 6 & 3 & 2007 & 6,3 & 11 & 5 & 49 \\
\hline 142 & 100,81 & $-0,54$ & 6 & 3 & 2007 & 5,3 & 20 & 10 & 8 \\
\hline 143 & 100,6 & $-0,58$ & 6 & 3 & 2007 & 5,3 & 20 & 17 & 37 \\
\hline 144 & 100,56 & $-1,1$ & 14 & 9 & 2007 & 5,3 & 35 & 5 & 46 \\
\hline 145 & 100,67 & $-0,47$ & 6 & 3 & 2007 & 5,2 & 20 & 16 & 2 \\
\hline 146 & 100,51 & $-0,72$ & 8 & 3 & 2007 & 5,2 & 20 & 22 & 39 \\
\hline 147 & 100,86 & $-0,91$ & 13 & 9 & 2007 & 5,2 & 35 & 13 & 40 \\
\hline 148 & 100,712 & $-0,54$ & 6 & 3 & 2007 & 5,03689 & 20 & 8 & 49 \\
\hline 149 & 100,738 & $-0,55$ & 6 & 3 & 2007 & 5,03689 & 20 & 16 & 23 \\
\hline 150 & 100,913 & $-0,399$ & 6 & 3 & 2007 & 5 & 20 & 12 & 53 \\
\hline 151 & 100,443 & $-1,03$ & 4 & 9 & 2009 & 4,83647 & 45,7 & 5 & 24 \\
\hline 152 & 100,775 & $-0,452$ & 6 & 3 & 2007 & 4,73626 & 20 & 8 & 13 \\
\hline 153 & 100,929 & $-0,611$ & 13 & 9 & 2007 & 4,73626 & 35 & 0 & 54 \\
\hline 154 & 100,661 & $-0,672$ & 6 & 3 & 2007 & 4,73626 & 20 & 20 & 14 \\
\hline 155 & 100,423 & $-0,759$ & 2 & 8 & 2007 & 4,73626 & 30 & 12 & 56 \\
\hline 156 & 101,009 & $-0,452$ & 6 & 3 & 2007 & 4,63605 & 20 & 15 & 22 \\
\hline 157 & 99,859 & 0,626 & 17 & 12 & 2006 & 5,8 & 30 & 21 & 39 \\
\hline 158 & 99,96 & 0,5 & 17 & 12 & 2006 & 5,4 & 30 & 21 & 54 \\
\hline 159 & 99,76 & 0,72 & 14 & 8 & 2008 & 5,3 & 35 & 9 & 8 \\
\hline 160 & 99,72 & 0,76 & 13 & 7 & 2008 & 5,3 & 10 & 3 & 56 \\
\hline 161 & 99,59 & 0,35 & 24 & 5 & 2008 & 5,3 & 35 & 17 & 50 \\
\hline
\end{tabular}




\begin{tabular}{|c|c|c|c|c|c|c|c|c|c|}
\hline \multirow{2}{*}{ No. } & \multirow{2}{*}{$\operatorname{Long}\left({ }^{\circ}\right)$} & \multirow{2}{*}{$\operatorname{Lat}\left(^{0}\right)$} & \multicolumn{3}{|c|}{ Date } & \multirow{2}{*}{$\begin{array}{l}\text { Mag. } \\
\left(\mathbf{M}_{\mathrm{w}}\right)\end{array}$} & \multirow{2}{*}{ depth $(\mathbf{k m})$} & \multicolumn{2}{|c|}{ time } \\
\hline & & & dt & $\mathbf{m n}$ & $\mathrm{yr}$ & & & hr & mn \\
\hline 162 & 99,56 & 0,06 & 3 & 1 & 2007 & 5,3 & 30 & 4 & 48 \\
\hline 163 & 100,09 & 0,57 & 19 & 12 & 2006 & 5,3 & 35,6 & 10 & 18 \\
\hline 164 & 100,28 & 0,95 & 31 & 3 & 2008 & 5,2 & 42,7 & 11 & 59 \\
\hline 165 & 99,8 & 0,78 & 19 & 5 & 2008 & 5,2 & 10 & 18 & 36 \\
\hline 166 & 99,74 & 0,35 & 8 & 1 & 2007 & 5,2 & 30 & 22 & 54 \\
\hline 167 & 100,23 & 0,55 & 18 & 12 & 2006 & 5,2 & 30 & 9 & 43 \\
\hline 168 & 100 & 0,05 & 17 & 12 & 2006 & 5,2 & 30 & 21 & 54 \\
\hline 169 & 99,98 & 0,46 & 17 & 12 & 2006 & 5,2 & 30 & 23 & 1 \\
\hline 170 & 99,69 & 0,51 & 17 & 12 & 2006 & 5,2 & 30 & 23 & 18 \\
\hline 171 & 100,112 & 0,864 & 9 & 5 & 2007 & 4,93668 & 7,6 & 18 & 30 \\
\hline 172 & 99,368 & 0,557 & 18 & 12 & 2006 & 4,83647 & 30 & 1 & 24 \\
\hline 173 & 99,958 & 0,504 & 17 & 12 & 2006 & 4,63605 & 30 & 21 & 54 \\
\hline 174 & 99,093 & 1,751 & 29 & 3 & 2005 & 5,5 & 23,4 & 6 & 16 \\
\hline 175 & 98,92 & 2,05 & 9 & 1 & 2006 & 5,3 & 13,9 & 22 & 25 \\
\hline 176 & 99,23 & 1,93 & 30 & 3 & 2005 & 5,3 & 30 & 2 & 20 \\
\hline 177 & 99,25 & 1,83 & 29 & 3 & 2005 & 5,3 & 30 & 10 & 51 \\
\hline 178 & 99,35 & 1,83 & 29 & 3 & 2005 & 5,3 & 30 & 10 & 34 \\
\hline 179 & 99,45 & 1,81 & 3 & 4 & 2005 & 5,3 & 30 & 2 & 0 \\
\hline 180 & 99,48 & 1,8 & 11 & 7 & 2005 & 5,3 & 30,9 & 3 & 19 \\
\hline 181 & 99,21 & 1,66 & 22 & 9 & 2005 & 5,3 & 30 & 14 & 13 \\
\hline 182 & 99,18 & 1,63 & 29 & 3 & 2005 & 5,3 & 30 & 22 & 17 \\
\hline 183 & 98,95 & 2,15 & 10 & 1 & 2006 & 5,2 & 30 & 4 & 55 \\
\hline 184 & 99,22 & 1,63 & 29 & 3 & 2005 & 5,2 & 30 & 22 & 14 \\
\hline 185 & 99,05 & 1,53 & 22 & 5 & 2007 & 5,2 & 30 & 5 & 6 \\
\hline 186 & 99,27 & 1,759 & 28 & 4 & 2007 & 4,9 & 23,9 & 19 & 14 \\
\hline 187 & 98,98 & 2,004 & 16 & 6 & 2005 & 4,83647 & 30 & 12 & 17 \\
\hline 188 & 99,175 & 1,661 & 12 & 11 & 2005 & 4,83647 & 30 & 20 & 34 \\
\hline 189 & 98,935 & 2,277 & 9 & 4 & 2005 & 4,73626 & 30 & 18 & 14 \\
\hline 190 & 98,933 & 1,894 & 16 & 6 & 2005 & 4,73626 & 30 & 12 & 37 \\
\hline 191 & 99,58 & 1,944 & 17 & 9 & 2005 & 4,63605 & 20 & 21 & 53 \\
\hline 192 & 99,221 & 1,872 & 11 & 7 & 2005 & 4,63605 & 30 & 3 & 23 \\
\hline 193 & 99,072 & 1,713 & 11 & 4 & 2005 & 4,63605 & 30 & 11 & 42 \\
\hline 194 & 99,155 & 1,671 & 29 & 3 & 2005 & 4,63605 & 30 & 19 & 4 \\
\hline 195 & 97,568 & 4,495 & 22 & 1 & 2003 & 5,83857 & 33 & 2 & 58 \\
\hline 196 & 97,439 & 4,717 & 27 & 12 & 2004 & 4,63605 & 30 & 9 & 31 \\
\hline 197 & 97,567 & 4,395 & 19 & 9 & 2003 & 4,83647 & 33 & 20 & 41 \\
\hline 198 & 97,43 & 4,58 & 5 & 4 & 2005 & 5,3 & 30 & 17 & 25 \\
\hline 199 & 97,62 & 4,72 & 25 & 3 & 2005 & 5,2 & 30 & 22 & 28 \\
\hline 200 & 97,646 & 4,619 & 13 & 9 & 2003 & 5,3 & 33 & 20 & 42 \\
\hline 201 & 97,737 & 4,505 & 22 & 1 & 2003 & 4,63605 & 33 & 5 & 59 \\
\hline 202 & 97,768 & 4,517 & 12 & 11 & 2003 & 4,63605 & 33 & 5 & 48 \\
\hline
\end{tabular}




\begin{tabular}{|c|c|c|c|c|c|c|c|c|c|}
\hline \multirow{2}{*}{ No. } & \multirow{2}{*}{ Long( $\left(^{\circ}\right)$} & \multirow{2}{*}{$\operatorname{Lat}\left({ }^{0}\right)$} & \multicolumn{3}{|c|}{ Date } & \multirow{2}{*}{$\begin{array}{l}\text { Mag. } \\
\left(\mathbf{M}_{\mathrm{w}}\right)\end{array}$} & \multirow{2}{*}{ depth $(\mathbf{k m})$} & \multicolumn{2}{|c|}{ time } \\
\hline & & & dt & $\mathbf{m n}$ & yr & & & hr & $\mathbf{m n}$ \\
\hline 203 & 97,77 & 4,37 & 3 & 2 & 2003 & 5,3 & 33 & 12 & 13 \\
\hline 204 & 97,81 & 4,23 & 26 & 12 & 2004 & 5,6 & 30 & 21 & 19 \\
\hline 205 & 97,811 & 4,233 & 26 & 12 & 2004 & 5,03689 & 30 & 21 & 19 \\
\hline 206 & 97,815 & 4,377 & 12 & 1 & 2005 & 5,03689 & 30 & 10 & 28 \\
\hline 207 & 105,16 & $-6,298$ & 15 & 1 & 2002 & 6,1 & 24,2 & 7 & 13 \\
\hline 208 & 105,205 & $-6,314$ & 15 & 1 & 2002 & 6,1 & 10 & 7 & 12 \\
\hline 209 & 105,59 & $-5,74$ & 15 & 1 & 2002 & 5,3 & 10 & 7 & 43 \\
\hline 210 & 105,68 & $-6,12$ & 26 & 2 & 2002 & 5,3 & 33 & 6 & 17 \\
\hline 211 & 104,88 & $-6,87$ & 21 & 3 & 2003 & 5,3 & 33 & 4 & 26 \\
\hline 212 & 104,536 & $-6,339$ & 28 & 10 & 2002 & 5,23731 & 35,2 & 20 & 37 \\
\hline 213 & 104,453 & $-5,964$ & 17 & 2 & 2002 & 5,2 & 45 & 10 & 23 \\
\hline 214 & 105,645 & $-6,607$ & 5 & 1 & 2003 & 5,2 & 33 & 16 & 16 \\
\hline 215 & 105,36 & $-5,67$ & 7 & 8 & 2004 & 5,3 & 43,9 & 19 & 9 \\
\hline 216 & 105,244 & $-6,157$ & 16 & 1 & 2002 & 5,03689 & 10 & 15 & 26 \\
\hline 217 & 105,627 & $-5,806$ & 4 & 7 & 2003 & 4,93668 & 33 & 14 & 0 \\
\hline 218 & 105,32 & $-7,04$ & 15 & 8 & 2004 & 5,3 & 50 & 18 & 48 \\
\hline 219 & 105,142 & $-6,252$ & 22 & 2 & 2002 & 4,93668 & 21,3 & 13 & 33 \\
\hline 220 & 105,702 & $-6,407$ & 11 & 10 & 2002 & 4,93668 & 33 & 6 & 6 \\
\hline 221 & 104,841 & $-5,866$ & 2 & 8 & 2004 & 4,93668 & 18,1 & 21 & 16 \\
\hline 222 & 105,203 & $-6,612$ & 15 & 7 & 2003 & 4,93668 & 33 & 1 & 22 \\
\hline 223 & 105,297 & $-6,167$ & 17 & 9 & 2003 & 4,83647 & 33 & 6 & 6 \\
\hline 224 & 105,229 & $-6,464$ & 26 & 1 & 2002 & 4,83647 & 33 & 3 & 26 \\
\hline 225 & 105,315 & $-5,739$ & 22 & 8 & 2004 & 4,73626 & 10 & 20 & 52 \\
\hline 226 & 105,273 & $-5,625$ & 2 & 8 & 2004 & 4,63605 & 20 & 20 & 57 \\
\hline 227 & 105,59 & $-5,878$ & 17 & 3 & 2002 & 4,73626 & 33 & 9 & 59 \\
\hline 228 & 105,165 & $-6,142$ & 22 & 2 & 2002 & 4,73626 & 15,5 & 13 & 52 \\
\hline 229 & 105,37 & $-6,158$ & 20 & 1 & 2002 & 4,73626 & 10 & 1 & 18 \\
\hline 230 & 105,253 & $-5,673$ & 29 & 7 & 2004 & 4,63605 & 18,5 & 0 & 19 \\
\hline 231 & 104,563 & $-5,569$ & 24 & 4 & 2004 & 4,73626 & 10 & 15 & 11 \\
\hline 232 & 104,515 & $-5,858$ & 16 & 9 & 2003 & 4,63605 & 33 & 0 & 31 \\
\hline 233 & 104,735 & $-5,915$ & 20 & 12 & 2003 & 4,63605 & 33 & 15 & 0 \\
\hline 234 & 104,988 & $-6,66$ & 15 & 1 & 2002 & 4,63605 & 10 & 21 & 4 \\
\hline 235 & 104,942 & $-6,805$ & 25 & 1 & 2003 & 4,63605 & 33 & 9 & 22 \\
\hline 236 & 96,405 & 4,284 & 20 & 8 & 1997 & 6 & 9,5 & 7 & 15 \\
\hline 237 & 97,09 & 3,99 & 27 & 9 & 1997 & 5,3 & 33 & 0 & 2 \\
\hline 238 & 96,314 & 4,135 & 17 & 9 & 1997 & 4,83647 & 20 & 4 & 16 \\
\hline 239 & 96,685 & 4,332 & 21 & 10 & 1997 & 4,63605 & 20 & 4 & 43 \\
\hline 240 & 95,759 & 4,929 & 15 & 2 & 1999 & 5,5 & 16 & 5 & 46 \\
\hline 241 & 96,06 & 4 & 23 & 10 & 1999 & 5,3 & 45,5 & 18 & 18 \\
\hline 242 & 95,8 & 4,69 & 21 & 2 & 1999 & 5,3 & 33 & 14 & 35 \\
\hline 243 & 96,46 & 4,318 & 8 & 12 & 1999 & 4,63605 & 33 & 14 & 15 \\
\hline
\end{tabular}




\begin{tabular}{|c|c|c|c|c|c|c|c|c|c|}
\hline \multirow{2}{*}{ No. } & \multirow{2}{*}{ Long( $\left(^{\circ}\right)$} & \multirow{2}{*}{$\operatorname{Lat}\left({ }^{0}\right)$} & \multicolumn{3}{|c|}{ Date } & \multirow{2}{*}{$\begin{array}{l}\text { Mag. } \\
\left(\mathbf{M}_{\mathrm{w}}\right)\end{array}$} & \multirow{2}{*}{ depth $(\mathbf{k m})$} & \multicolumn{2}{|c|}{ time } \\
\hline & & & dt & $\mathbf{m n}$ & yr & & & hr & $\mathbf{m n}$ \\
\hline 244 & 95,869 & 4,737 & 10 & 3 & 2000 & 5,5 & 12 & 21 & 32 \\
\hline 245 & 96,43 & 3,91 & 4 & 3 & 2000 & 5,1 & 33 & 18 & 13 \\
\hline 246 & 105,123 & $-6,031$ & 10 & 8 & 1985 & 5,6 & 22,3 & 4 & 12 \\
\hline 247 & 105,11 & $-6,64$ & 1 & 4 & 1988 & 5,3 & 33 & 8 & 48 \\
\hline 248 & 104,609 & $-6,712$ & 2 & 3 & 1987 & 5,23731 & 33 & 22 & 34 \\
\hline 249 & 104,675 & $-6,116$ & 26 & 2 & 1987 & 5,23731 & 35 & 12 & 16 \\
\hline 250 & 105,291 & $-5,987$ & 20 & 3 & 1986 & 5,23731 & 25 & 21 & 0 \\
\hline 251 & 105,56 & $-6,76$ & 24 & 11 & 1986 & 5,2 & 33 & 8 & 8 \\
\hline 252 & 105,471 & $-6,735$ & 5 & 4 & 1988 & 5,1371 & 33 & 1 & 21 \\
\hline 253 & 104,802 & $-6,002$ & 9 & 9 & 1985 & 5,1371 & 35 & 10 & 36 \\
\hline 254 & 104,826 & $-6,367$ & 3 & 9 & 1987 & 5,03689 & 33 & 8 & 33 \\
\hline 255 & 105,408 & $-6,039$ & 14 & 9 & 1986 & 5,03689 & 25 & 3 & 38 \\
\hline 256 & 104,673 & $-6,179$ & 22 & 4 & 1986 & 4,93668 & 33 & 21 & 25 \\
\hline 257 & 104,905 & $-5,93$ & 21 & 2 & 1987 & 4,93668 & 35 & 12 & 55 \\
\hline 258 & 104,719 & $-5,325$ & 22 & 10 & 1987 & 4,93668 & 33 & 1 & 0 \\
\hline 259 & 105,144 & $-6,642$ & 20 & 10 & 1986 & 4,83647 & 50 & 23 & 53 \\
\hline 260 & 104,236 & $-5,712$ & 3 & 1 & 1986 & 4,73626 & 33 & 7 & 53 \\
\hline 261 & 104,403 & $-5,694$ & 29 & 12 & 1985 & 4,73626 & 33 & 4 & 22 \\
\hline 262 & 104,271 & $-5,94$ & 10 & 1 & 1986 & 4,63605 & 25 & 14 & 27 \\
\hline 263 & 93,848 & 9,068 & 25 & 10 & 1970 & 6,4 & 24,6 & 15 & 9 \\
\hline 264 & 94,1 & 9,16 & 27 & 12 & 1970 & 5,4 & 33 & 4 & 50 \\
\hline 265 & 93,639 & 9,671 & 25 & 9 & 1972 & 5,33752 & 30 & 11 & 30 \\
\hline 266 & 93,991 & 9,249 & 26 & 12 & 1970 & 5,33752 & 16,5 & 10 & 2 \\
\hline 267 & 94,067 & 9,088 & 28 & 12 & 1971 & 5,23731 & 30 & 12 & 45 \\
\hline 268 & 94,042 & 9,071 & 25 & 10 & 1970 & 5,03689 & 28,9 & 15 & 21 \\
\hline 269 & 94,043 & 9,167 & 25 & 10 & 1970 & 4,93668 & 31,5 & 22 & 8 \\
\hline 270 & 93,839 & 9,145 & 26 & 12 & 1970 & 4,93668 & 14,1 & 10 & 15 \\
\hline 271 & 94,117 & 9,12 & 25 & 10 & 1970 & 4,93668 & 15 & 22 & 20 \\
\hline 272 & 93,985 & 9,064 & 25 & 10 & 1970 & 4,93668 & 10 & 17 & 53 \\
\hline 273 & 94,07 & 9,045 & 25 & 10 & 1970 & 4,93668 & 12,9 & 17 & 50 \\
\hline 274 & 94,132 & 9,07 & 25 & 10 & 1970 & 4,83647 & 15 & 15 & 42 \\
\hline 275 & 93,586 & 8,648 & 2 & 7 & 1967 & 6,1 & 6,5 & 7 & 3 \\
\hline 276 & 93,671 & 8,704 & 19 & 7 & 1968 & 5,8 & 26,2 & 4 & 56 \\
\hline 277 & 94 & 8,7 & 9 & 3 & 1968 & 5,6 & 33 & 0 & 46 \\
\hline 278 & 93,8 & 8,5 & 2 & 7 & 1967 & 5,6 & 33 & 14 & 19 \\
\hline 279 & 93,86 & 8,74 & 2 & 4 & 1969 & 5,4 & 33 & 12 & 36 \\
\hline 280 & 94,1 & 8,7 & 8 & 3 & 1968 & 5,4 & 33 & 23 & 8 \\
\hline 281 & 93,8 & 8,6 & 2 & 7 & 1967 & 5,4 & 33 & 18 & 36 \\
\hline 282 & 93,84 & 8,81 & 18 & 7 & 1968 & 5,3 & 33 & 17 & 43 \\
\hline 283 & 93,995 & 8,695 & 9 & 3 & 1968 & 5,23731 & 13 & 0 & 45 \\
\hline 284 & 93,787 & 8,981 & 19 & 7 & 1968 & 4,83647 & 32,4 & 6 & 7 \\
\hline
\end{tabular}




\begin{tabular}{|c|c|c|c|c|c|c|c|c|c|}
\hline \multirow{2}{*}{ No. } & \multirow{2}{*}{ Long $\left({ }^{\circ}\right)$} & \multirow{2}{*}{$\operatorname{Lat}\left({ }^{0}\right)$} & \multicolumn{3}{|c|}{ Date } & \multirow{2}{*}{$\begin{array}{l}\text { Mag. } \\
\left(\mathbf{M}_{\mathbf{w}}\right)\end{array}$} & \multirow{2}{*}{ depth (km) } & \multicolumn{2}{|c|}{ time } \\
\hline & & & dt & $\mathbf{m n}$ & yr & & & hr & mn \\
\hline 285 & 94,178 & 8,737 & 19 & 11 & 1968 & 4,83647 & 25,1 & 22 & 48 \\
\hline 286 & 93,764 & 8,517 & 2 & 7 & 1967 & 4,83647 & 28,4 & 14 & 9 \\
\hline 287 & 93,652 & 8,901 & 18 & 7 & 1968 & 4,73626 & 26,8 & 17 & 20 \\
\hline 288 & 93,74 & 8,843 & 19 & 7 & 1968 & 4,73626 & 25 & 16 & 42 \\
\hline 289 & 96,236 & 5,079 & 12 & 4 & 1967 & 6,7 & 17,5 & 4 & 51 \\
\hline 290 & 96,333 & 5,273 & 12 & 4 & 1967 & 5,6 & 15 & 5 & 11 \\
\hline 291 & 96,333 & 5,097 & 22 & 4 & 1967 & 5,53794 & 15,5 & 13 & 7 \\
\hline 292 & 96,64 & 5,12 & 12 & 4 & 1967 & 5,5 & 22 & 18 & 47 \\
\hline 293 & 96,678 & 5,211 & 12 & 4 & 1967 & 5,43773 & 15 & 19 & 33 \\
\hline 294 & 96,61 & 5,12 & 14 & 4 & 1967 & 5,4 & 34 & 12 & 5 \\
\hline 295 & 96,398 & 4,379 & 25 & 6 & 1969 & 5,33752 & 10 & 7 & 24 \\
\hline 296 & 96,196 & 4,864 & 5 & 6 & 1969 & 5,23731 & 23,5 & 10 & 45 \\
\hline 297 & 96,428 & 5,259 & 13 & 4 & 1967 & 5,23731 & 15 & 8 & 25 \\
\hline 298 & 96,445 & 5,402 & 12 & 4 & 1967 & 5,1371 & 35 & 5 & 18 \\
\hline 299 & 96,395 & 5,253 & 14 & 8 & 1967 & 5,1371 & 15 & 6 & 41 \\
\hline 300 & 96,614 & 5,357 & 12 & 4 & 1967 & 5,1371 & 15 & 6 & 3 \\
\hline 301 & 96,74 & 5,145 & 21 & 4 & 1967 & 5,1371 & 15 & 16 & 28 \\
\hline 302 & 95,813 & 5,077 & 5 & 12 & 1968 & 5,03689 & 15 & 9 & 1 \\
\hline 303 & 96,657 & 5,147 & 28 & 4 & 1967 & 5,03689 & 15 & 16 & 14 \\
\hline 304 & 96,46 & 5,229 & 15 & 5 & 1967 & 4,93668 & 15 & 18 & 50 \\
\hline 305 & 96,451 & 4,421 & 10 & 2 & 1970 & 4,73626 & 10 & 21 & 0 \\
\hline
\end{tabular}




\section{Code to calculate $\mathbf{C}(\mathbf{l})$}

import pandas as pd

from math import cos,sin,acos,degrees,radians

df1 = pd.read_excel("D2_3_2014.xlsx",sheet_name='Sheet1')

$\mathrm{N}=\operatorname{int}(\mathrm{df} 1 . \operatorname{loc}[0, " \operatorname{count} "])$

jumlah $=0$

maximal $=0$

minimal $=999$

$\operatorname{arrM=}[]$

$\operatorname{arrl}=[]$

\#print (int(N))

for $\mathrm{i}$ in range $(\mathrm{N})$ :

for $\mathrm{j}$ in range( $\mathrm{N})$ :

if $\mathrm{i} !=\mathrm{j}$ :

$\mathrm{m}=\operatorname{acos}(\cos (\operatorname{radians}(\mathrm{df} 1 . \operatorname{loc}[\mathrm{i}, " \mathrm{lat} "])) *$

$\cos (\operatorname{radians}(\mathrm{df} 1 . \operatorname{loc}[\mathrm{j}$, "lat"] $))+\sin (\operatorname{radians}(\mathrm{df1}$.loc[i,"lat"]))

* $\sin (\operatorname{radians}(\mathrm{df1} 1 \mathrm{loc}[\mathrm{j}, " \mathrm{lat} "])) *$

$\cos (\operatorname{radians}((\mathrm{df} 1 . \operatorname{loc}[\mathrm{i}, "$ long"] $)$ - (df1.loc[j,"long"] $))))$

arrM.append(m)

jumlah=jumlah +1

if $\mathrm{m}>=$ maximal:

maximal $=\mathrm{m}$

else:

maximal $=$ maximal

if $\mathrm{m}<=$ minimal: 


$$
\text { minimal }=\mathrm{m}
$$

else:

$$
\text { minimal }=\text { minimal }
$$

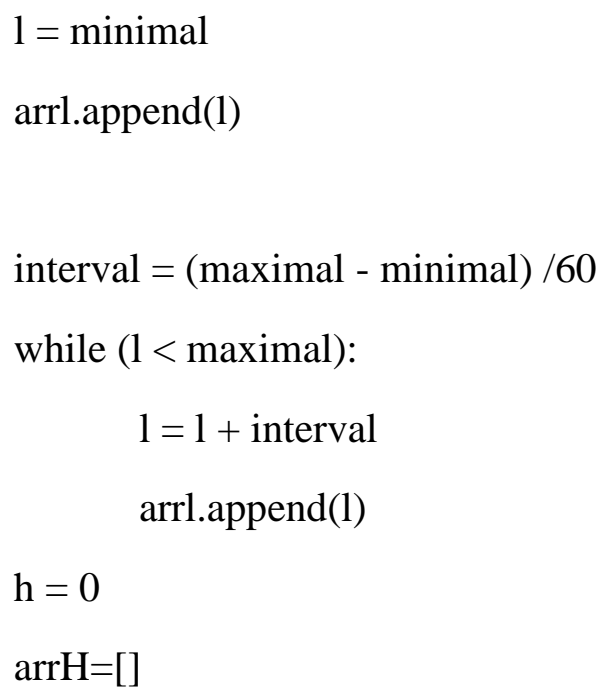

for $\mathrm{i}$ in range(len(arrl)):

$$
\mathrm{j} \mathrm{H}=0
$$

for $\mathrm{j}$ in range(len(arrM)):

$$
\begin{aligned}
& H=\operatorname{arrl}[i]-\operatorname{arrM}[j] \\
& \text { if }(H>=0) \text { : } \\
& \qquad H=1
\end{aligned}
$$

else:

$$
\begin{array}{r}
\mathrm{H}=0 \\
\mathrm{jH}=\mathrm{jH}+\mathrm{H}
\end{array}
$$

$\operatorname{arrH} . \operatorname{append}(\mathrm{jH})$

$$
\operatorname{arrCL}=[]
$$

for $\mathrm{i}$ in range(len(arrH)):

$$
\begin{aligned}
& \mathrm{x}=1 /(\mathrm{N} * * 2) * \operatorname{arrH}[\mathrm{i}] \\
& \operatorname{arrCL} . \operatorname{append}(\mathrm{x})
\end{aligned}
$$


$\mathrm{cl}=\left\{\right.$ 'cl': $\left.\operatorname{arrCL},{ }^{\prime} l ': \operatorname{arrl}\right\}$

$\mathrm{df}=\mathrm{pd} \cdot$ DataFrame $($ data $=\mathrm{cl})$

writer $=$ pd.ExcelWriter('out.xlsx')

df.to_excel(writer,'Sheet1')

writer.save() 
3. Earthquake distribution and double log. Curve of $I$ and C(I)

Nicobar 2014

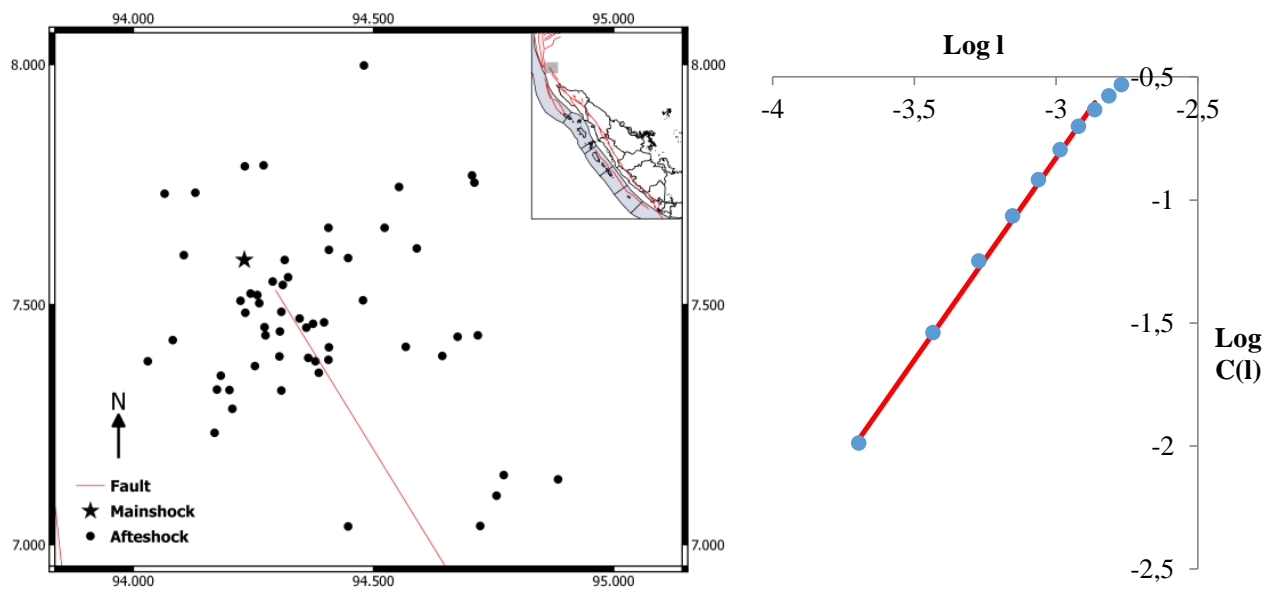

Aceh Jaya 2013
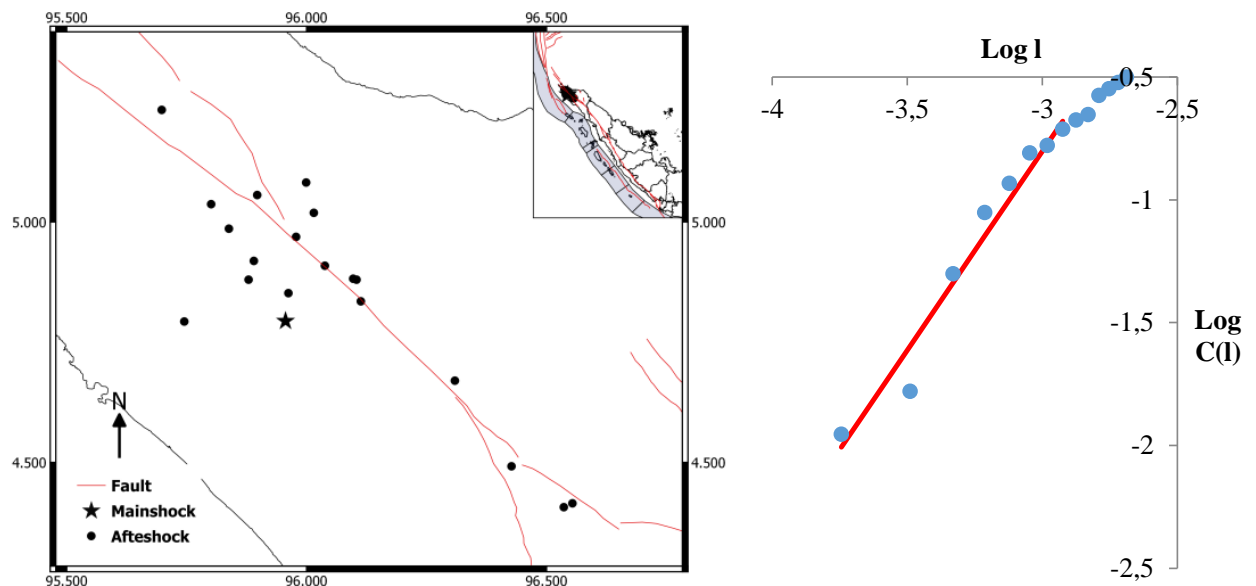

Tapanuli 2008
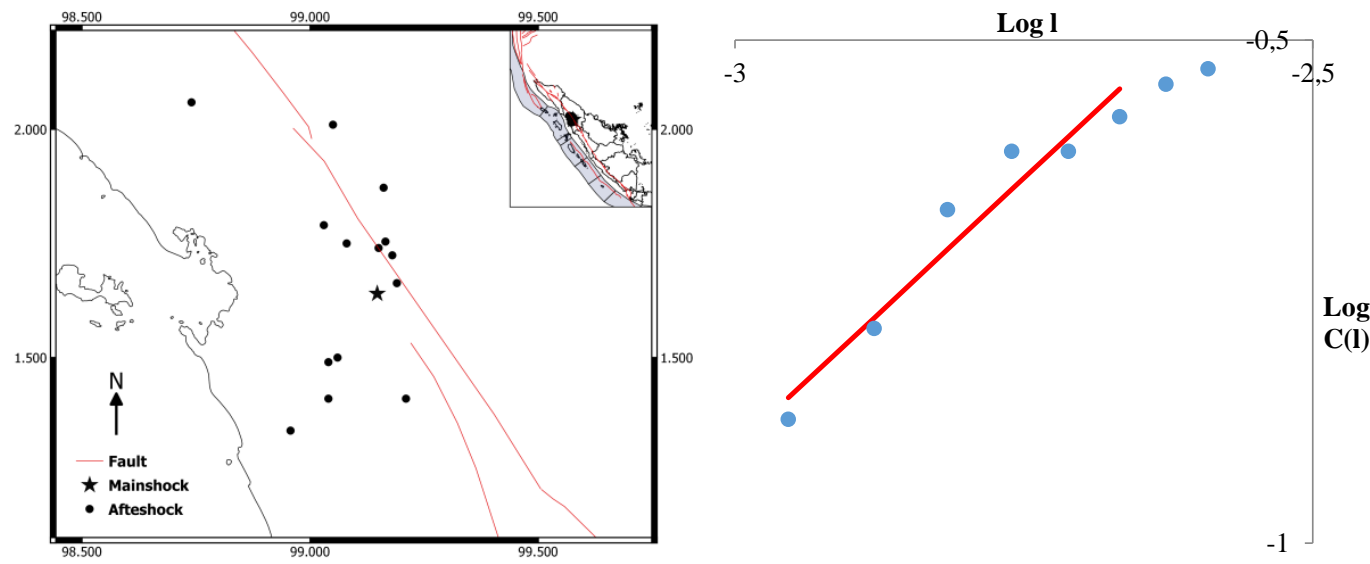
Tanah Datar 2007
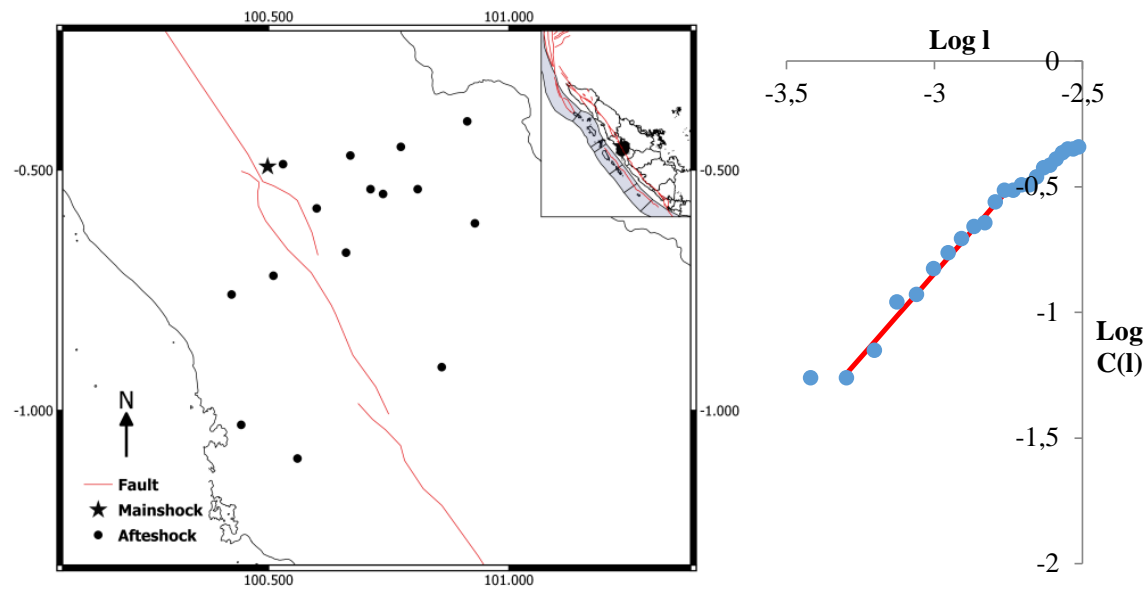

\section{Mandailingnatal 2006}
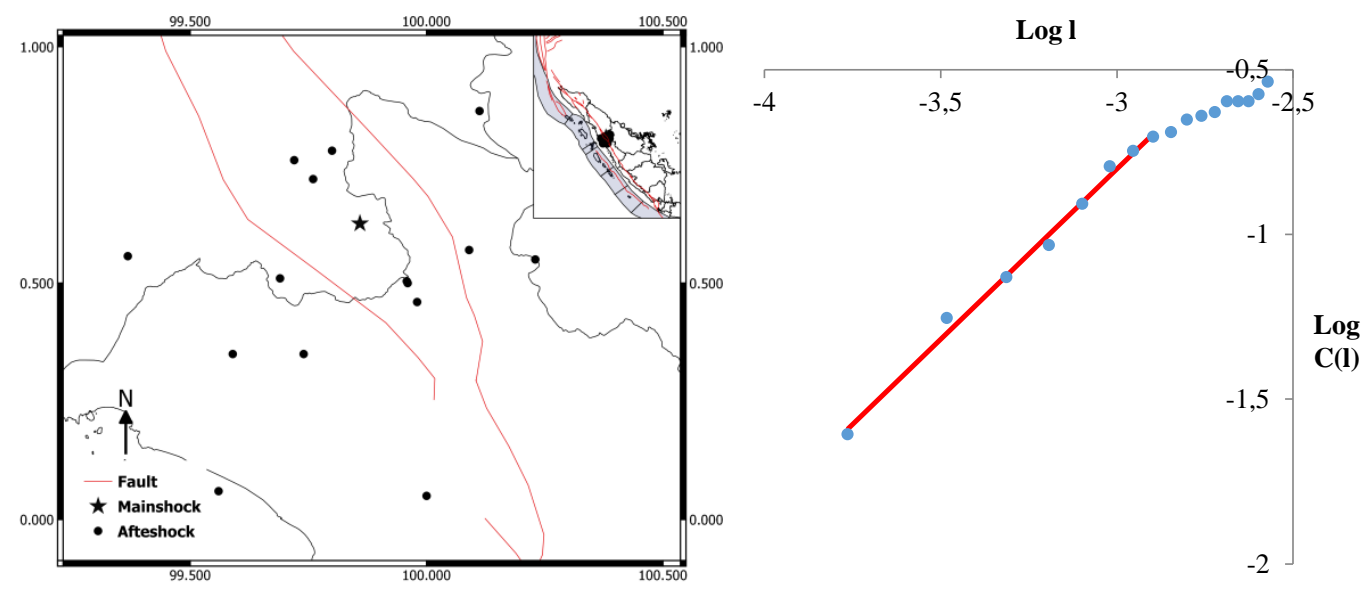

Tapanuli 2005
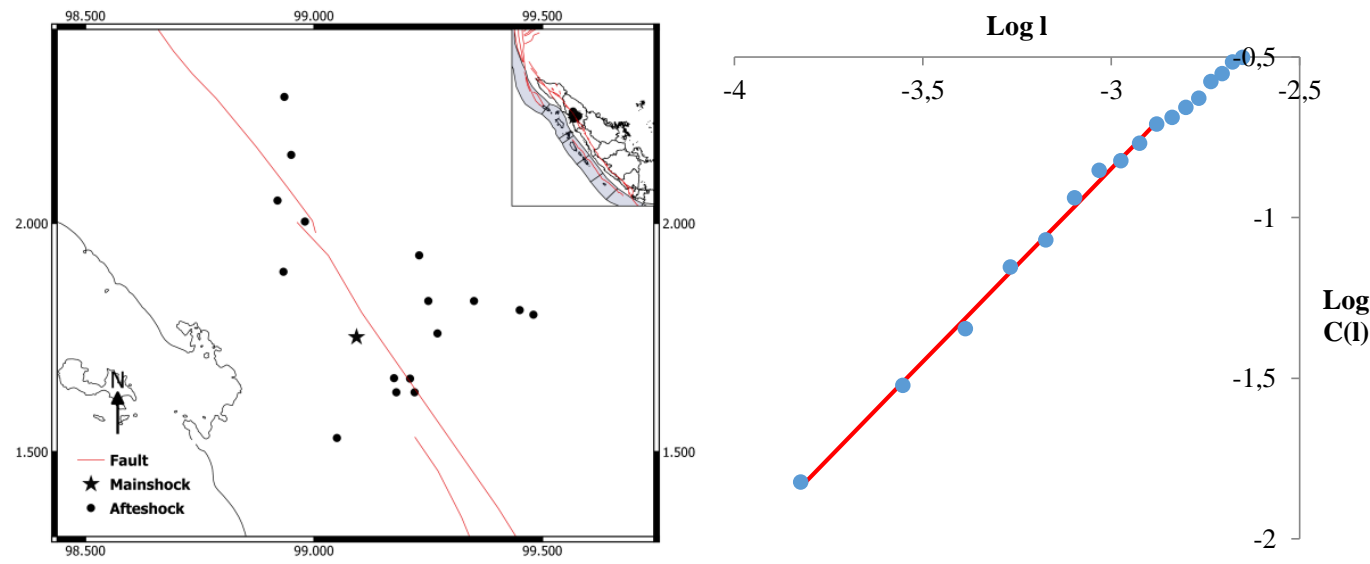
Aceh Timur 2003
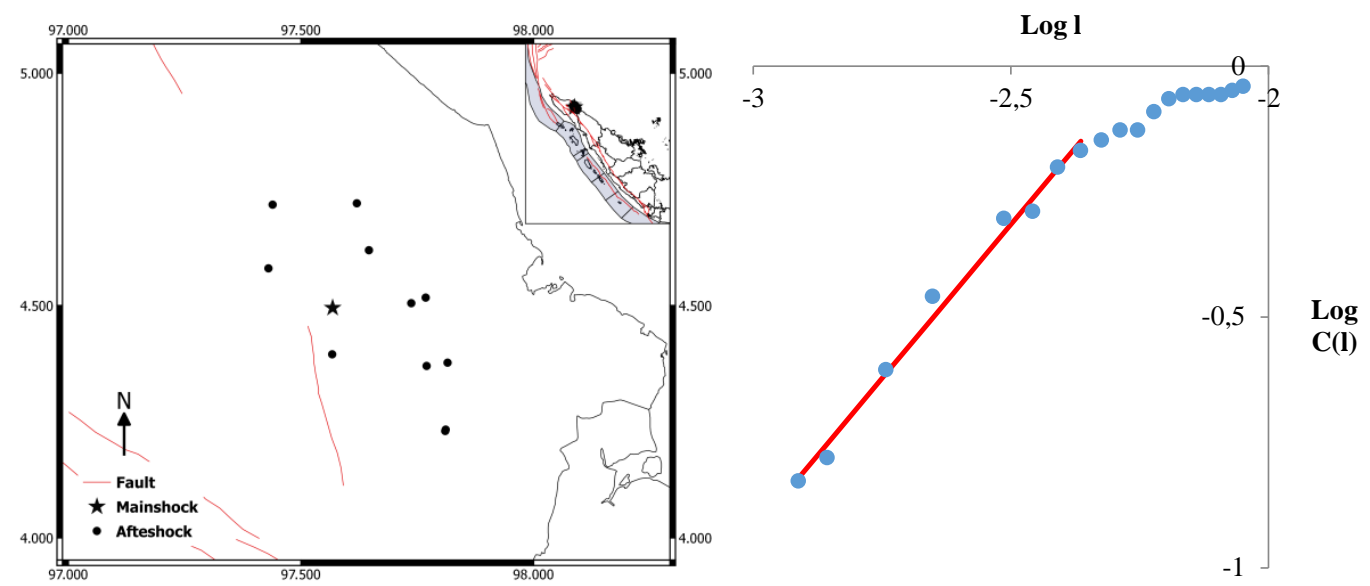

Selat Sunda 2002

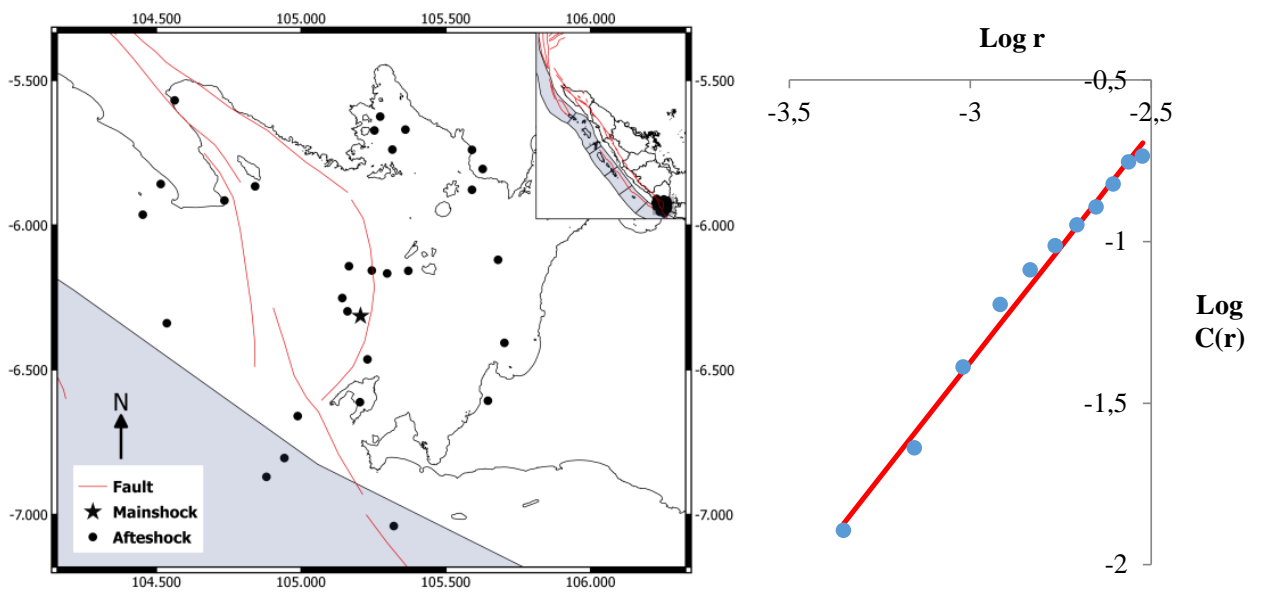

Naganraya 1997
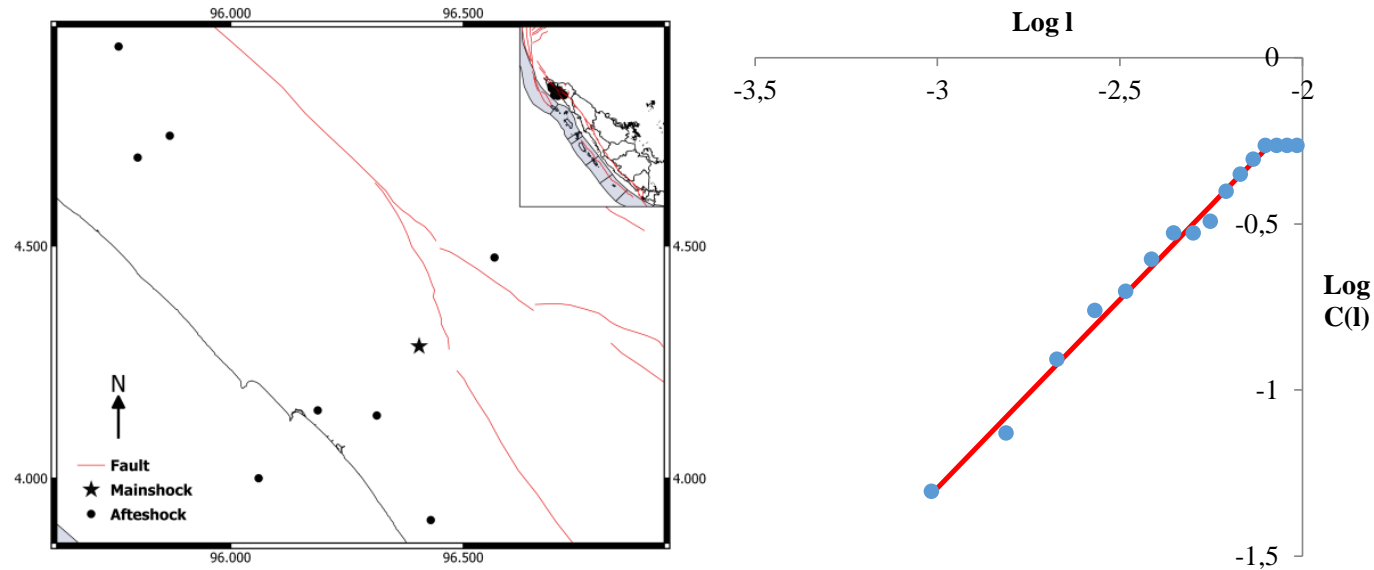


\section{Nikobar 1996}
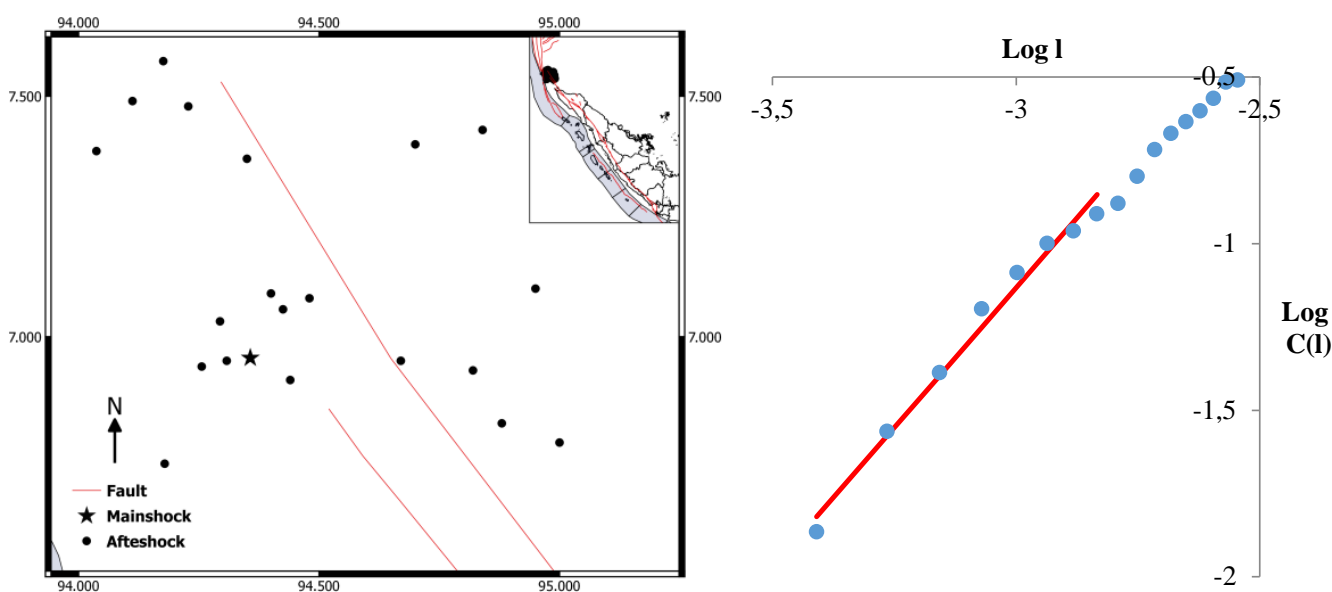

\section{Selat Sunda 1985}

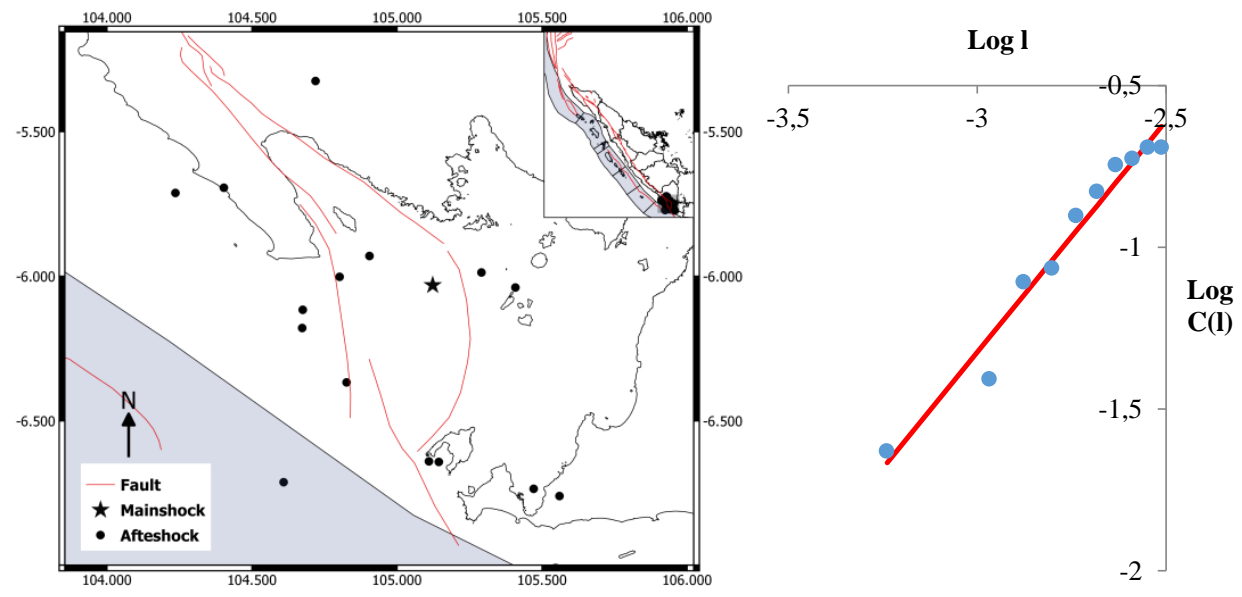

\section{Nikobar 1970}

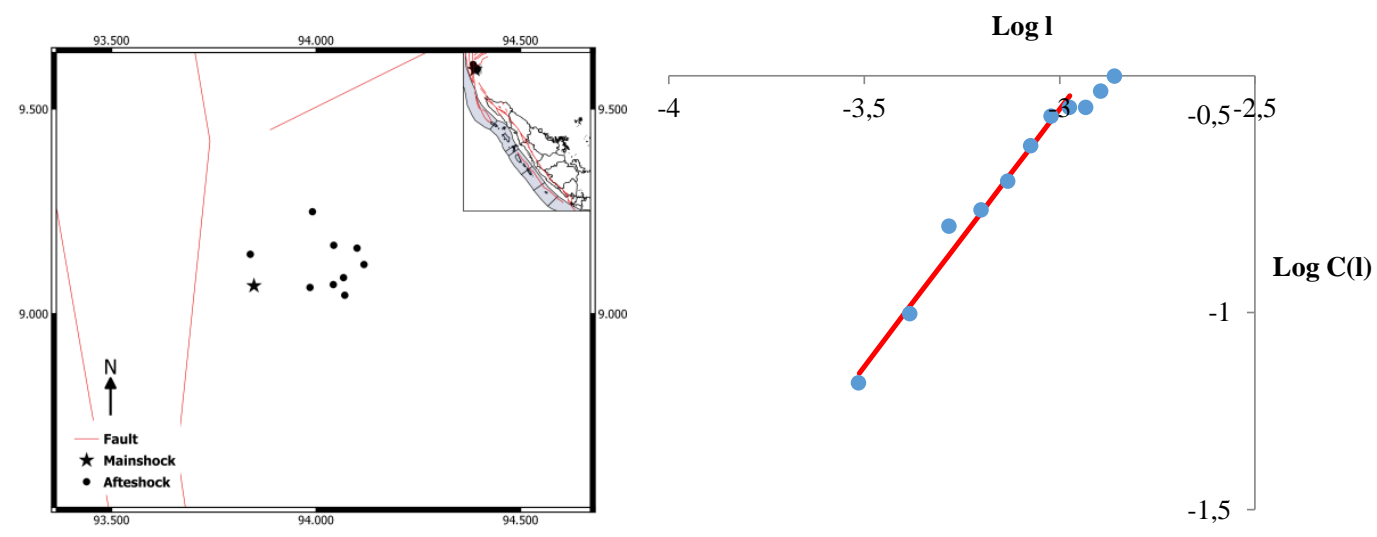


Nikobar 1967
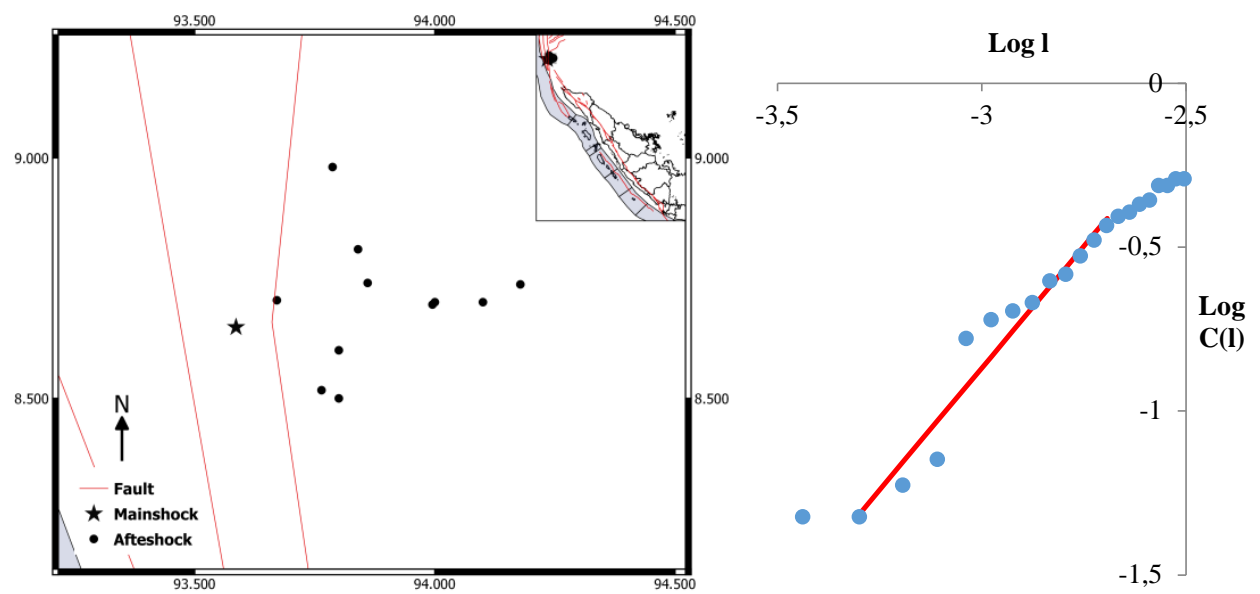

Pidie 1967
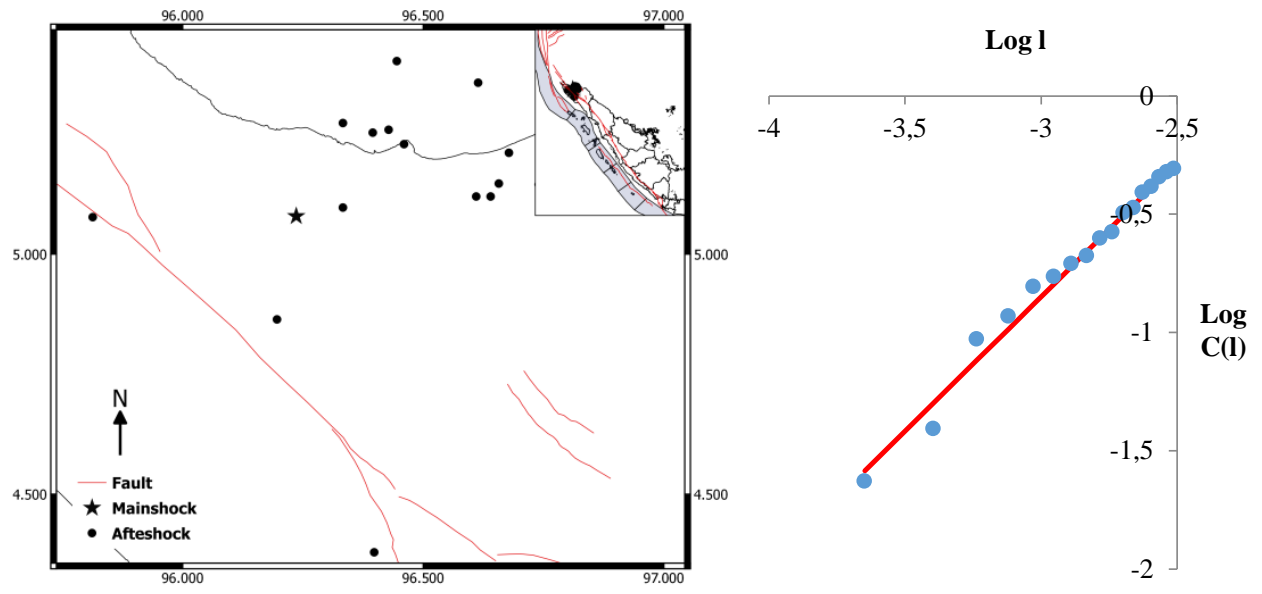
4. Active fault and double log curve of $\mathrm{N}$ and $\mathbf{r}$

Nicobar 2014
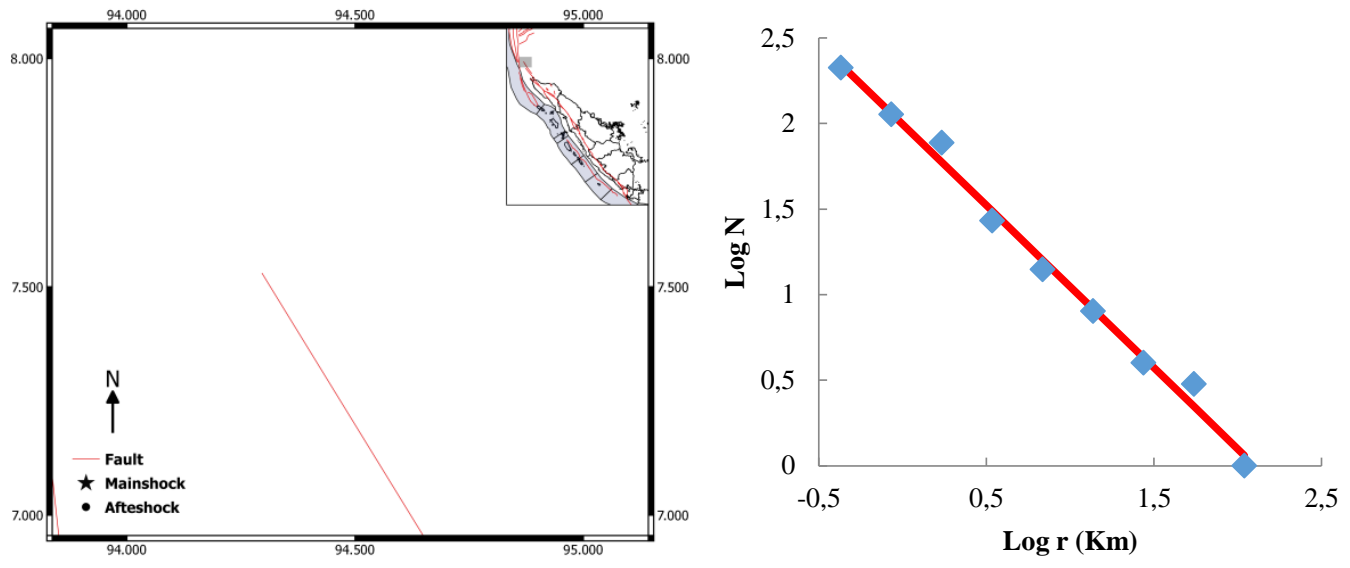

Aceh Jaya 2013
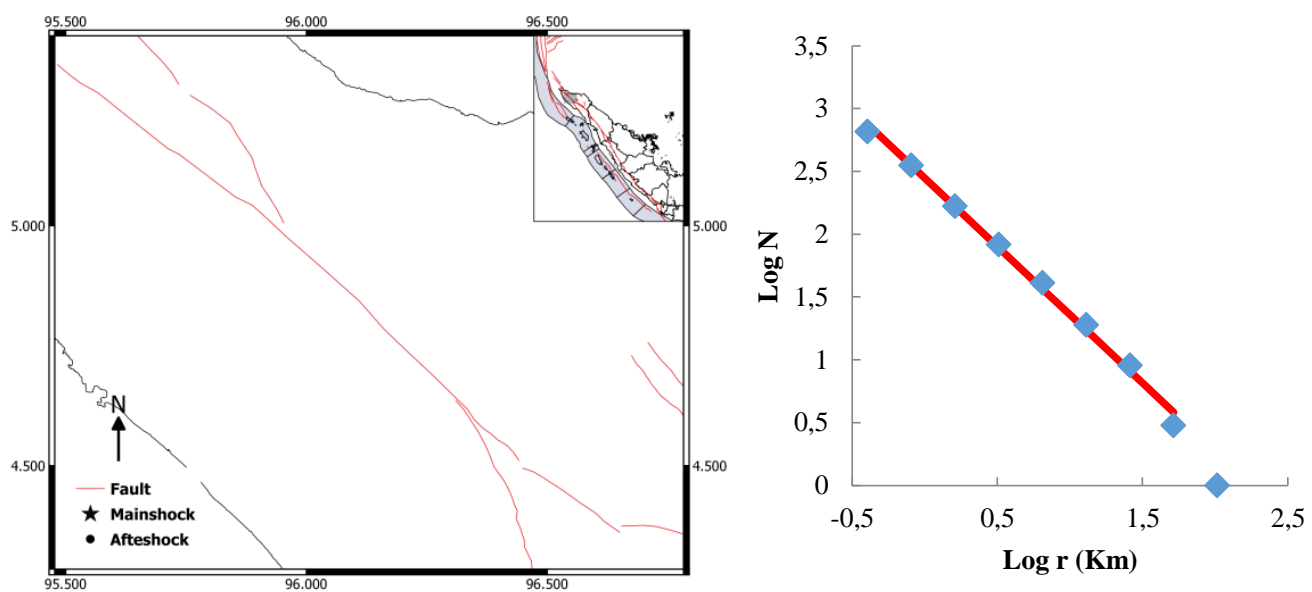

Tapanuli 2008
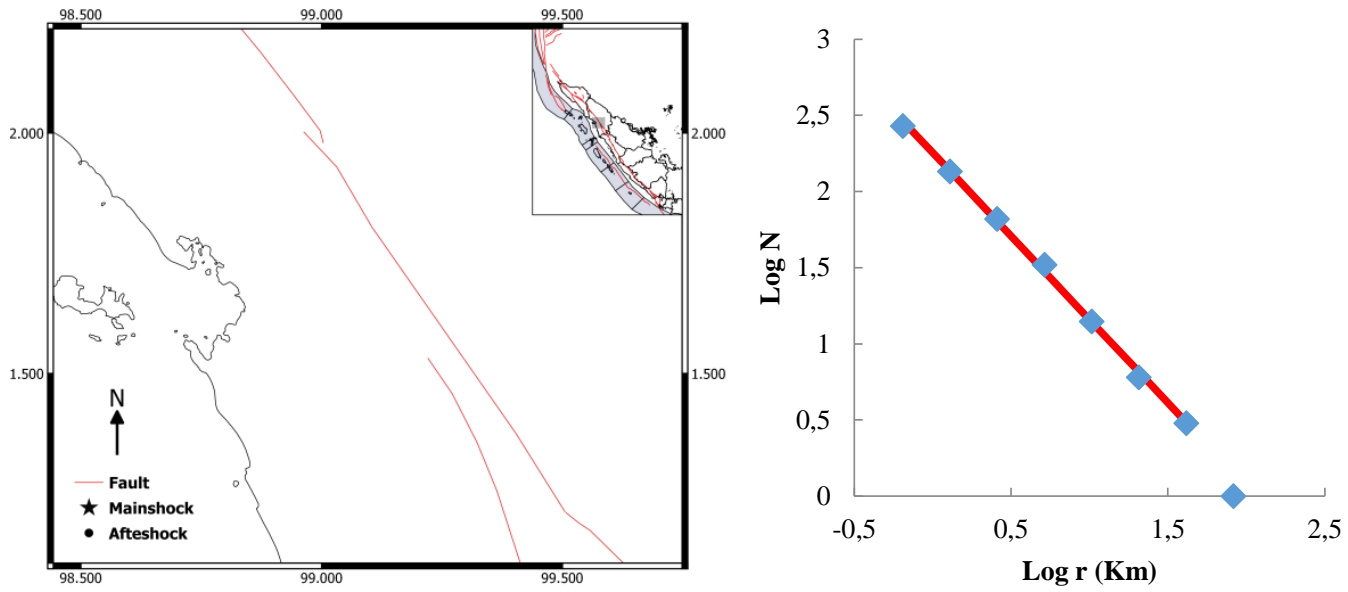

Tanah Datar 2007 

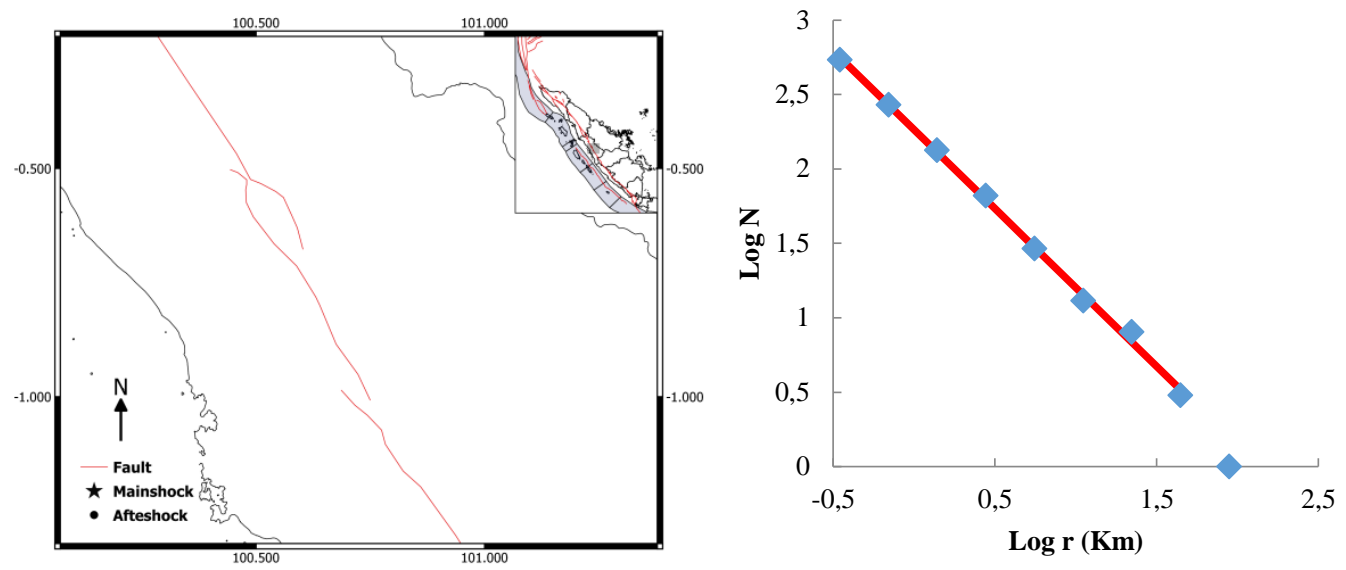

\section{Mandailingnatal 2006}
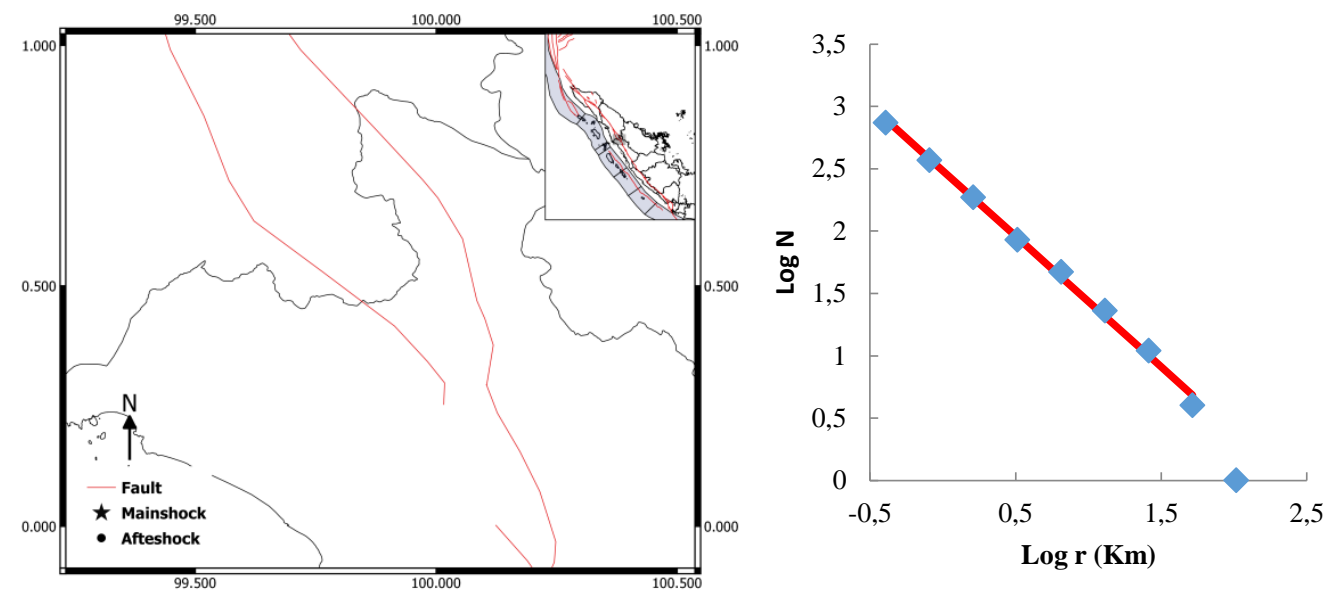

Tapanuli 2005
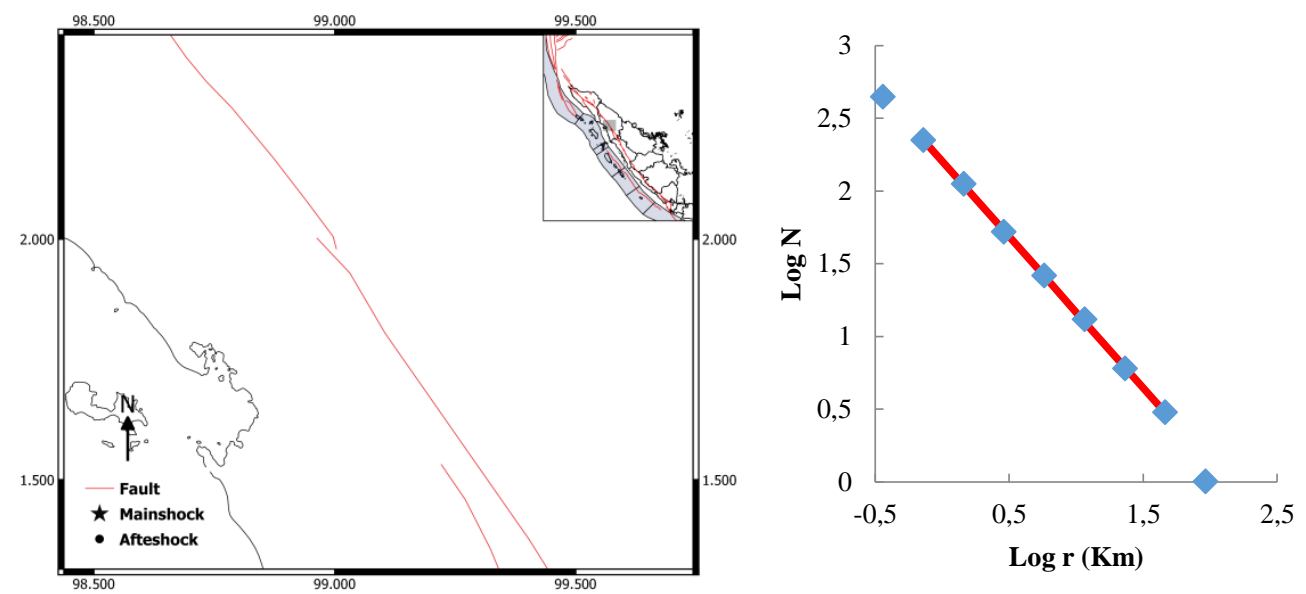

Aceh Timur 2003 

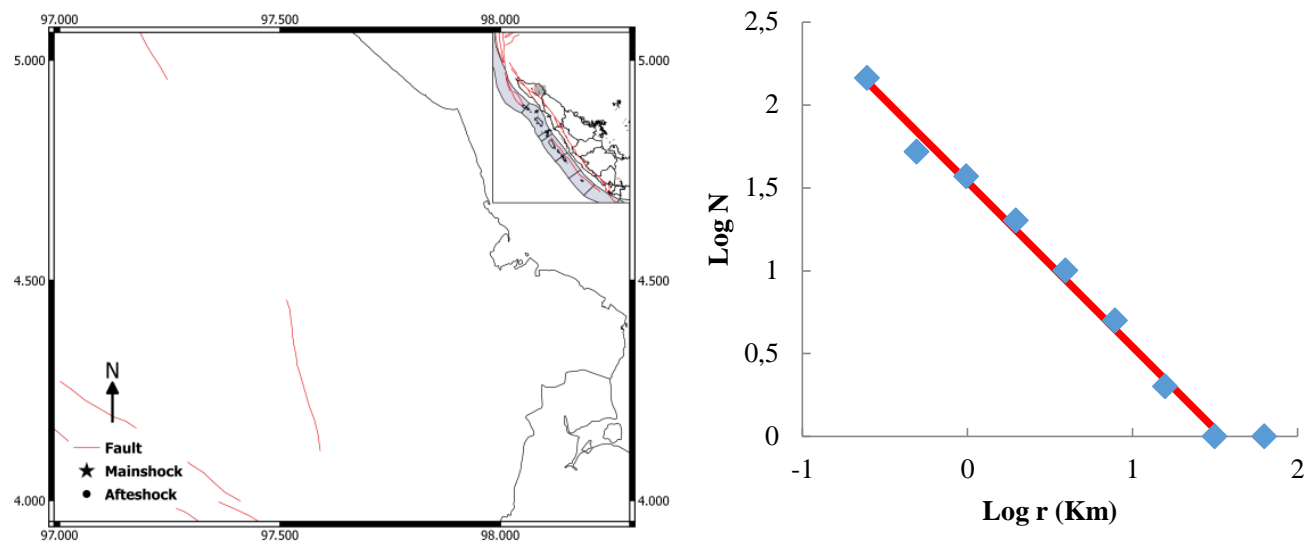

Selat Sunda 2002
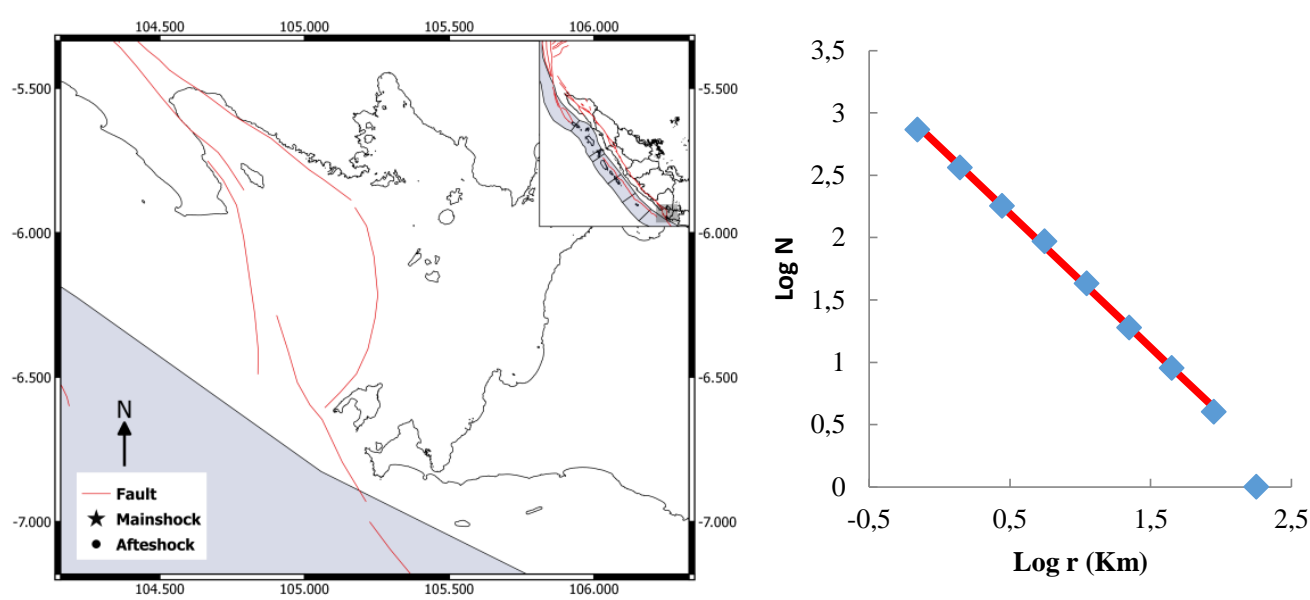

\section{Naganraya 1997}
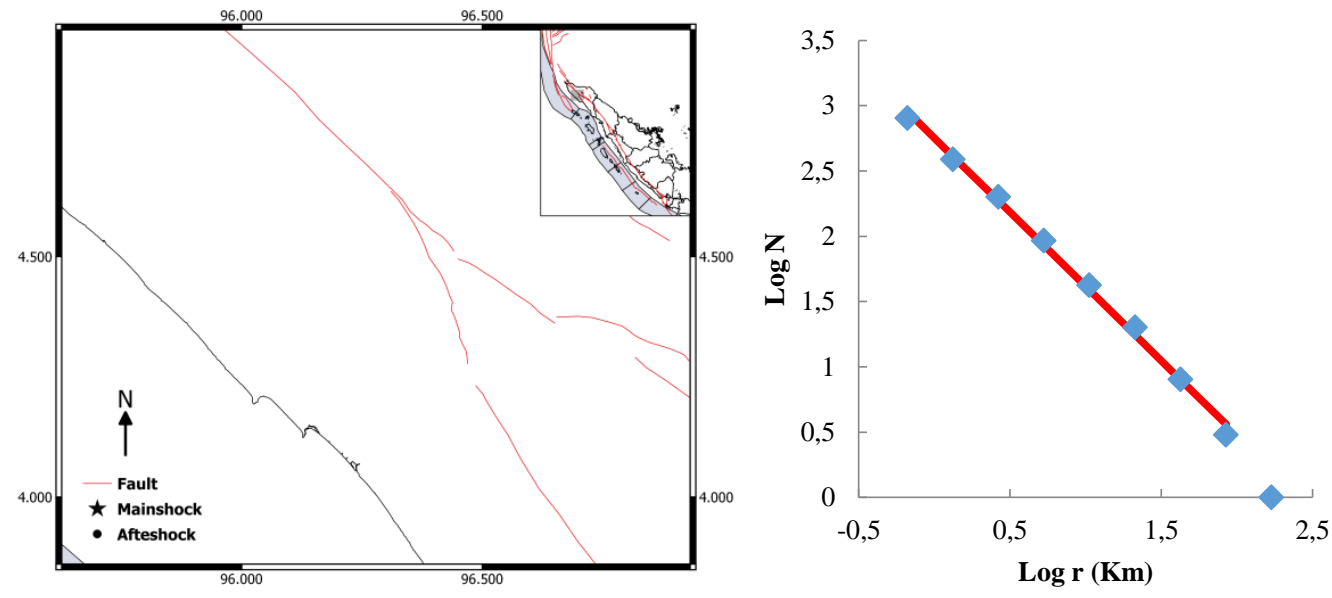

Nikobar 1996 

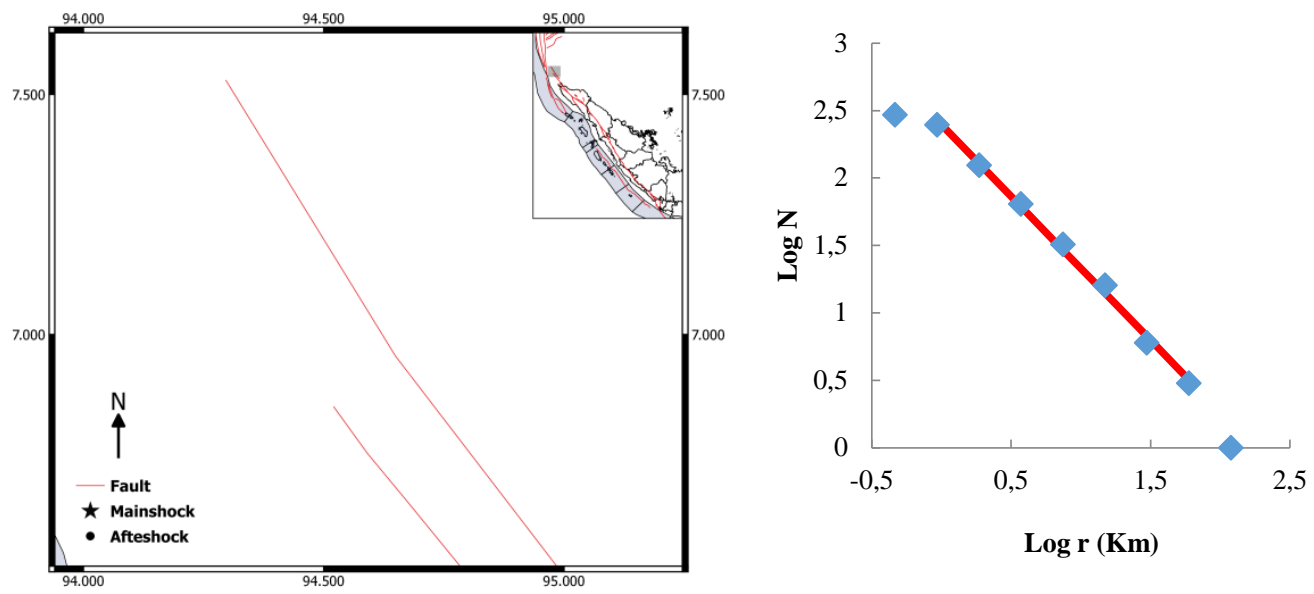

\section{Selat Sunda 1985}
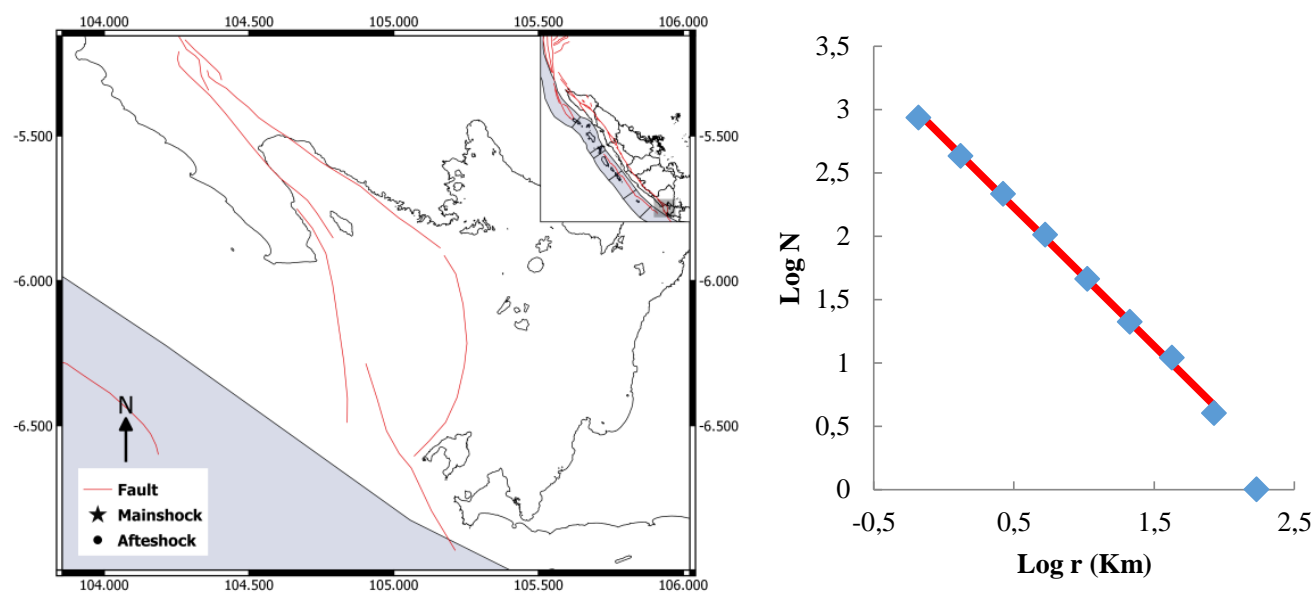

\section{Nikobar 1970}
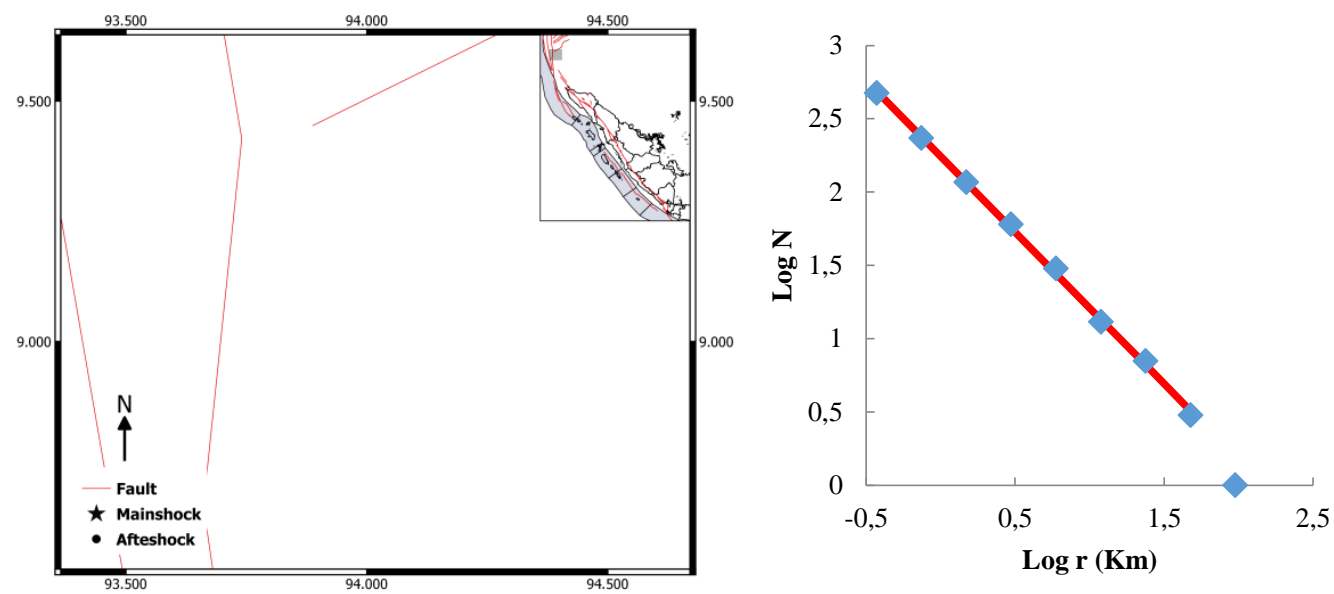

Nikobar 1967 

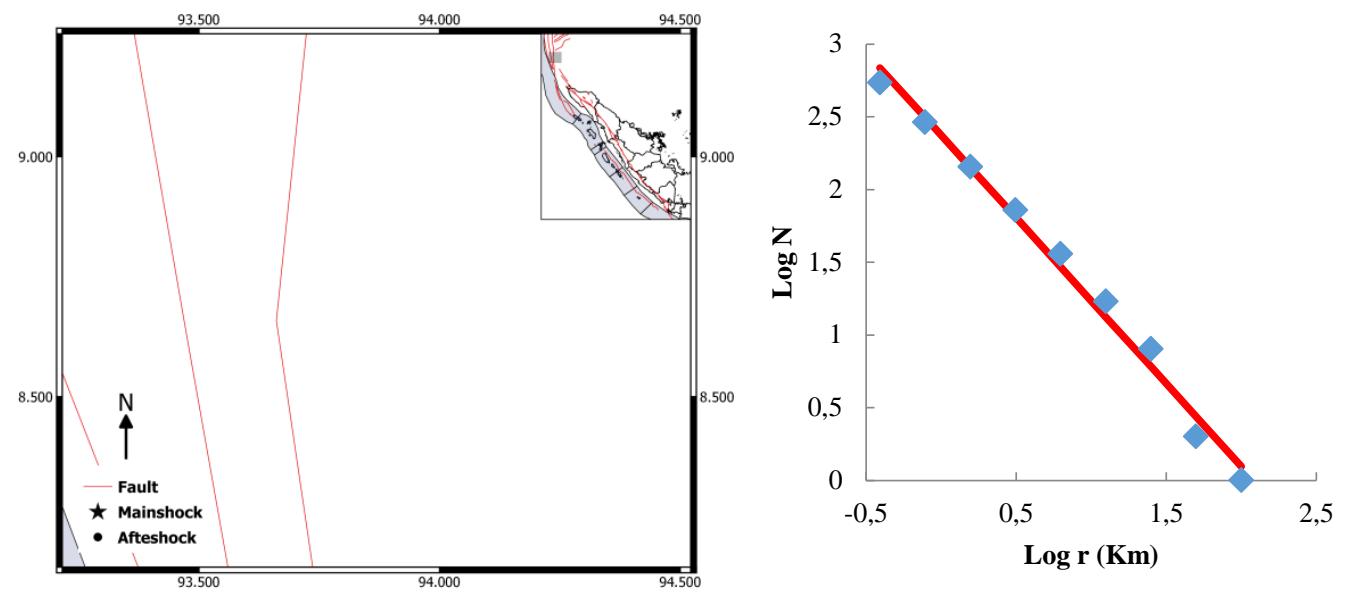

Pidie 1967
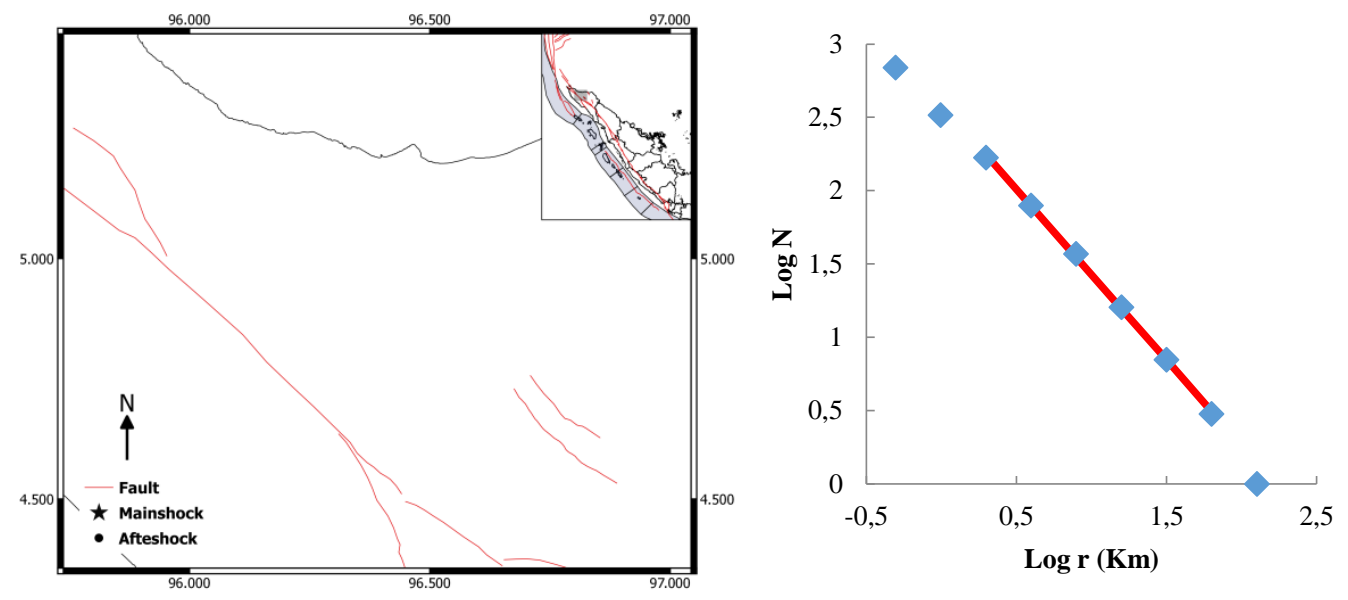